\title{
NK cells acquire epigenetic memory of LPS-induced systemic inflammation
}

Orhan Rasid ${ }^{1,2,}$, Christine Chevalier ${ }^{1}$, Tiphaine Camarasa ${ }^{1}$, Catherine Fitting ${ }^{2}$, Jean-Marc Cavaillon ${ }^{2}$, Melanie Anne Hamon ${ }^{1, *}$

${ }^{1}$ G5 Chromatine et Infection, Institut Pasteur, Paris, France

${ }^{2}$ Unité Cytokines \& Inflammation, Institut Pasteur, Paris, France

* Corresponding authors 


\begin{abstract}
Natural killer cells are unique mediators of innate immunity, and as such, an attractive target for immunotherapy. Following viral infection, NK cells display immune memory properties, defined by heightened responses to re-stimulation, an expansion of specific NK cell sup-populations and a protective role against re-infection. However, similar memory to bacterial infection or systemic inflammation, and the molecular mechanisms behind NK cell memory remain elusive. Here we show that following LPS-induced endotoxemia in mice, NK cells acquire cell-intrinsic memory properties as displayed by an amplified production of IFN $\gamma$ upon secondary stimulation. NK cell memory is acquired even under the post-endotoxemic suppressive environment and is detectable for at least 9 weeks. Furthermore, we define an epigenetic mechanism essential for NK cell memory, where an H3K4me1marked latent enhancer is uncovered at the ifng locus. Chemical inhibition of histone methyltransferase activity erased the enhancer and prevented NK cells from acquiring memory. Thus, NK cells develop memory to LPS during endotoxemia, in a histone methylation-dependent manner, which ensures a heightened response to secondary stimulation and confers protection against bacterial infection.
\end{abstract}




\section{INTRODUCTION}

In recent years, accumulating evidence of memory responses mediated by innate immune cells have blurred the boundaries between innate and adaptive immunity $(1,2)$. Monocytes and macrophages have been described to mediate a type of innate immune memory, termed trained immunity (3). Briefly, this refers to the long-lasting altered responsiveness of cells to a secondary stimulation, with either lower (tolerized) or higher (trained) inflammatory responses, involving epigenetic modifications and metabolic rewiring(4). While trained immunity in phagocytes is non-specific and results in a general hyper or hypo-responsiveness to re-stimulation, other innate cells such as NK cells can exhibit a high degree of antigen specificity in a secondary response $(3,5)$. NK cells have been shown to display memory properties to viral infection, cytokine stimulation or hapten-induced contact-hypersensitivity, but evidence for bacterial infection remains scarce(5). The specificity of the recall response together with proliferation of specific receptor-defined sub-populations make NK cell memory more akin to classical adaptive memory mediated by $\mathrm{T} \operatorname{cells}(\emptyset)$.

NK cell memory was demonstrated in several in vivo models of viral infection, particularly mouse cytomegalovirus (MCMV), where the murine Ly49H receptor, which specifically recognize the MCMV m157 glycoprotein, defines the population of responsive NK cells that acquire memory(7). In humans infected with CMV the NKG2C receptor was involved in ensuring NK cell responses to specific viral peptides(8). At the molecular level, NK cell memory to CMV has been correlated with modified chromatin states $(9)$. Mouse and human memory-like NK cells have modulated DNA methylation levels, which are notably reduced at the ifng/IFNG gene locus(10), and more accessible chromatin at effector genes as assessed by ATAC-Seq(11). However, whether these or other epigenetic marks are important for establishing and maintaining memory, remains to be determined.

A common feature of all NK cell memory models is their dependence on an initial inflammatory response with several key cytokines implicated as critical factors for NK cell memory acquisition(6). Systemic inflammation, such as sepsis or severe trauma, represents one of the most complex paradigms in immunology and involves an overzealous inflammatory response that often represents a lifethreatening condition(12). NK cells are key players and one of the main drivers of systemic inflammation during the acute phase, but following, they are severely incapacitated, similarly to 
monocytes and T cells(13-15). Specifically, NK cells have impaired cytotoxicity and cytokine production in mouse models and human sepsis patients(16-19). Altogether, these immune consequences are known as the "immunosuppressive" phase of systemic inflammation(20), a state of immune dysfunction that is thought to persist beyond the acute event, causing poor quality of life and increased susceptibility to infection(21). However, while short-term consequences on the immune system are fairly well explored, the long-term effects remain understudied. Indeed, the long-term status of NK cells after systemic inflammation remains to be explored and could have important implications, given their well-known role as critical effectors in viral and bacterial infections(22).

Herein, we used an endotoxemia model to explore the lasting impact of systemic inflammation on NK cells. We found that NK cells acquire cell-intrinsic memory properties, demonstrated by increased IFN $\gamma$ production upon re-challenge, up to 9 weeks after endotoxemia. Strikingly, systemic inflammation revealed a latent enhancer at the ifng locus in memory NK cells and blocking this, along with histone methylation, prevented acquisition of memory. Thus, we report that despite the suppressive immune environment that follows systemic inflammation, NK cells retain memory properties that are epigenetically encoded and help protect mice against bacterial infection. 


\section{RESULTS}

\section{NK cells are responsive after endotoxemia and acquire memory properties despite being in a suppressive environment}

In order to assess the long-term effects of systemic inflammation on NK cells, we used an endotoxemia model (10 mg/kg LPS, i.p.) causing acute inflammation but low mortality, with full clinical recovery by day 7 (Fig. 1a,b,c). Indeed, mice exhibited transient weight loss, clinical signs of inflammation and limited morbidity. Using this model, we had previously shown that NK cells are systemically activated over a 48-hour acute period(23). In the current study, we focused on the impact of endotoxemia on NK cells, 14 days after LPS injection. First, we phenotypically defined NK cells at 14 days after endotoxemia (full gating strategy in fig. S1). We found no significant differences in NK cell percentages or total numbers in the spleen of mice that received PBS injections as controls (D14PBS) or experienced endotoxemia 14 days prior (D14LPS) (Fig. 1d,e). Furthermore, although we found some variations in NK cell phenotype (fig. S1b,c,d), such differences did not correlate with an altered baseline of IFN $\gamma$ expression detected upon ex-vivo staining in D14LPS mice compared to D14PBS (fig. S1e). Therefore, altogether these data indicate that 14 days after endotoxemia, while NK cells acquire slight phenotypical differences, they return to a resting state, similar to that of naïve cells.

We next tested the responsiveness of NK cells from post-endotoxemia mice in vitro both within the environment of the spleen and separately as purified cells. We first stimulated total spleen cells with LPS and observed significantly reduced IFN $\gamma$ positive NK cell percentages from D14LPS samples as compared to D14PBS controls, as well as reduced total IFN $\gamma$ secretion in supernatants (Fig. 1f,h,i). In correlation with the lower levels of NK cell activation in D14LPS mice, we found a significant increase in percentages of regulatory $\mathrm{T}$ cells (Tregs) and accumulation of immature myeloid cells in these mice as compared to D14PBS controls (fig. S2). Therefore, the immune environment in the spleen 14 days after systemic inflammation is suppressive and affects NK cells, which is in agreement with previous reports(24-26). Interestingly, stimulation with IL-12+IL-18 stimulation, targeted NK cell activators, induced similar levels of NK cells response between D14PBS and D1LPS (Fig. 1g,h,i). These results were surprising and suggested that while post-endotoxemia spleen cells cannot activate NK cells in 
response to LPS, the intrinsic responsiveness of NK cells, at least to IL-12+IL-18, was not altered 14 days after endotoxemia.

To further study the response of NK cells independently of the splenic environment, we stimulated enriched NK cells $(\sim 80 \%)$ in vitro. We had previously shown that isolated NK cells respond to LPS stimulation in vitro if co-stimulated with the cytokines IL-15+IL-18, which do not induce sustained activation on their own(16). In agreement, while overnight stimulation with IL-15+IL-18 induced no detectable response, IL-12+IL-18 stimulation induced a sustained IFN $\gamma$ production. However, no differences were observed between control D14PBS and D14LPS NK cells in terms of IFN $\gamma+$ percentages or total IFN $\gamma$ production in supernatants (Fig. $1 \mathrm{k}, 1, \mathrm{~m}$ ), as we had observed when stimulating total spleen cells. In contrast, upon stimulation with IL-15+IL-18+LPS, we found that D14LPS NK cells had significantly higher percentages of IFN $\gamma+$ cells compared to control D14PBS NK cells (Fig. 1j,1). The higher responsiveness of D14LPS NK cells was confirmed by measuring total levels of secreted IFN $\gamma$, as well as GM-CSF, in cell culture supernatants (Fig. 1m,n). Altogether, these results indicate that following systemic inflammation, NK cells have a significantly increased responsiveness to LPS in vitro, a memory-like property.

Since our in vitro stimulations are performed on enriched NK cells, we cannot exclude a possible effect of contaminants such as myeloid or T cells. To exclude this possibility, we performed the same experiments as above, but mixed enriched ( 80\%) naïve CD45.1 NK cells with CD45.2 D14PBS or D14LPS NK cells, which were highly purified ( $98 \%)$. In this way, each well containing D14PBS or D14LPS NK cells has an internal control of naïve NK cells, which are subjected to the same influence of possible contaminants. Cocultured naïve and D14PBS NK cells responded similarly to IL-15+IL18+LPS stimulation, as expected (fig. S1f,g). Furthermore, naïve cells cocultured with D14LPS NK cells reached slightly higher levels of IFN $\gamma$ expression compared to D14PBS NK cells but these were significantly surpassed by the cocultured D14LPS NK cells (fig. S1g,h). Therefore, these results argue against a major effect of accessory cell contaminants in our in vitro stimulation system and strongly support the cell-intrinsic nature of memory-like responses observed in D14LPS NK cells. Notably, the unaltered production of IFN $\gamma$ in response to cytokine stimulation would suggest that changes in NK cell 
reactivity after endotoxemia do not simply reflect a state of hyperresponsiveness. These observations are in agreement to what was previously shown for memory NK cells(27). Taken together, our results show that 2 weeks following endotoxemia, NK cells are still responsive and have acquired cell-intrinsic memory properties, while being in a suppressive environment that restrains their activation during a secondary challenge with LPS.

\section{Endotoxemia-induced NK cell memory is detectable in vivo and is cell-intrinsic}

We further explored the memory responses of NK cells in vivo upon LPS challenge. In order to compare D14LPS NK cells to naïve counterparts in the same immune environment, we used an adoptive transfer model in which naïve congenic NK cells were transferred into post-endotoxemic mice (Fig. 2a). We transferred naïve CD45.1 NK cells into CD45.2 mice that had been treated with PBS or LPS 13 days prior. On day 14, recipient mice were re-challenged with PBS or LPS and NK cell responses in the spleen were assessed 6 hours later by flow-cytometry. Transferred naïve NK cells were readily identified by CD45.1 staining and their IFN $\gamma$ expression was quantifiable (Fig. 2b). Importantly, control PBS injection induced no detectable IFN $\gamma$ in neither the endogenous or transferred NK cell populations, further demonstrating that 14 days after endotoxemia, NK have returned to a resting state (Fig. 2c). LPS re-challenge induced significant expression of IFN $\gamma$ by endogenous NK cells as well as transferred naïve NK cells in D14PBS mice (Fig. 2d, left). Since both endogenous and transferred NK cells responded at similar levels, the transfer procedure itself had no influence on IFN $\gamma$ response. In D14LPS mice, the percentages of IFN $\gamma+$ NK cells were significantly lower as compared to those from D14PBS mice (Fig. 2d), as expected given the suppressive environment we described. Strikingly, endogenous D14LPS NK cells had significantly higher percentages of IFN $\gamma+$ than transferred naïve NK cells (Fig. 2d, right). These results demonstrate that NK cell memory acquired after endotoxemia results in increased responsiveness to re-stimulation in vivo even under the control of the suppressive environment induced by systemic inflammation.

The Ly49H and NKG2C receptors have both been implicated in mediating NK cell memory responses to MCMV and CMV, respectively $(7,28)$, and we observed significant differences in the proportions of 
NK cell subpopulations defined by these receptors after endotoxemia (fig. S1c). We thus investigated whether subpopulations defined by these receptors might be involved in mediating memory in our model. We compared populations defined by NKG2A/C/E and Ly49H in both D14PBS and D14LPS mice, and found no difference in the percentages of IFN $\gamma+$ cells upon LPS re-challenge between positive or negative populations (fig. S3). These results suggest that neither of these subpopulations is involved in the observed results, thus, we conducted the rest of our study using the bulk NK cell population.

To explore memory NK cell function independently of the surrounding immunological environment, we turned to another adoptive transfer system in which we can evaluate intrinsic NK cell properties. For this, we transferred D13PBS or D13LPS NK cells into naïve congenic mice one day before re-challenge with PBS or LPS (Fig. 2e). Thus, CD45.2 D14PBS or D14LPS NK cells were compared to naïve endogenous CD45.1 NK cells 6 hours after PBS or LPS challenge (Fig. 2f). PBS injection revealed no differences in basal IFN $\gamma$ expression between transferred CD45.2 and endogenous naive CD45.1 NK cells, suggesting that both D14PBS or D14LPS NK cells remain in a resting state even outside the suppressive environment of the post-endotoxemia spleen (Fig. 2g). Upon LPS injection, a high percentage of IFN+ cells is detected both in control transferred D14PBS NK cells and endogenous naïve NK cells, at similar values (Fig. 2h, left). Strikingly, significantly higher percentages of IFN $\gamma+$ cells were detected among the transferred D14LPS NK cells compared to the respective endogenous NK cells (Fig. 2h, right). These results firmly demonstrate that NK cells intrinsically acquire memory features 14 days following systemic inflammation and this is independent of the surrounding immune environment.

\section{Endotoxemia-induced NK cell memory persists for at least 9 weeks}

The lasting potential is an important feature of a memory response, which we explored 9 weeks after endotoxemia. For this we tested the responsiveness of NK cells by performing adoptive transfer schemes similar to those in Fig. 2a and e, but 9 weeks after PBS or LPS injection (We9PBS/LPS). Remarkably, memory properties of NK cells were retained at this moment. Indeed, endogenous We9LPS NK cells responded with more IFN $\gamma$ than transferred naïve NK cells, despite the suppressive environment, which was still present at this time (Fig. 3a), albeit less restrictive than at day 14 . Additionally, transferred We9LPS NK cells still surpassed endogenous NK cells in IFN $\gamma+$ percentages 
in naïve recipient mice (Fig. 3b). We also performed in vitro stimulations of enriched NK cells and observed We9LPS NK cells still being significantly more responsive to IL-15+IL-18+LPS that We9PBS NK cells (Fig. 3c,d). Therefore, post-endotoxemia NK cells retain memory properties, characterized by an increased IFN $\gamma$ production to LPS challenge, a phenotype that persists for at least 9 weeks.

\section{Memory cells derive from NK cells that experienced endotoxemia}

To investigate the origin of D14LPS NK cells and whether these cells are the same (or descendants of the same) cells present at the time of endotoxemia, or new cells that matured and arrived in the spleen after systemic inflammation, we made use of the adoptive transfer system described in Fig. 4a. In this system, traceable congenic NK cells are transferred into recipient mice 1 day prior to endotoxemia and are followed through day 14. An additional transfer of naïve NK cells is performed 7 days after endotoxemia in order to have control naïve cells at day 14 in the same recipient mouse. Thus, we have a model in which a defined population of pre-transferred CD45.1 NK cells will have been exposed to the same control (D14PBS) or inflammatory (D14LPS) conditions as the endogenous CD45.1/2 NK cells, and a post-transferred population of naïve CD45.2 NK cells that will serve as additional naïve controls. Using this model, we re-challenged D14PBS and D14LPS CD45.1/2 mice with PBS or LPS and assessed NK cell IFN $\gamma$ expression. Due to the time period between the pre-transfer and the response readout, and the potential for NK cells plasticity of NK cells into type 1 innate lymphoid cells (ILC1s) in inflammatory and immunosuppressive environments like tumors(29), we increased the stringency of our gating strategy. We introduced an additional marker to our panel, and defined our NK cells as CD3 ${ }^{-}$ NK $1.1^{+} \mathrm{CD}_{49 \mathrm{~b}^{+}}$(Fig. 4b), thereby excluding interference of ILC1s in these experiments. Pre-transferred CD45.1 and post-transferred CD45.2 NK cells were readily detectable. Upon PBS injection, we observed no difference in basal IFN $\gamma$ expression by any NK cell population in D14PB or LPS mice (Fig. 4c). Furthermore, upon LPS injection into D14PBS mice, we observed similar percentages of IFN $\gamma+$ cells among the pre-transferred D14PBS CD45.1, endogenous CD45.1/2 D14PBS, and post-transferred naïve CD45.2 NK cell populations (Fig. 4d, left and fig. S4a), demonstrating that in the absence of prior inflammation, transferred and endogenous cell populations behave similarly. However, upon LPS injection into D14LPS mice, we observe an increase in percentages of IFN $\gamma+$ cells in endogenous 
D14LPS CD45.1/2 NK cells compared to post-transferred naïve CD45.2 NK cells (Fig. 4d, right and fig. S4b). Most interestingly, the pre-transferred D14LPS CD45.1 NK cells also showed significantly higher IFN $\gamma+$ percentages compared to post-transferred naïve CD45.2 NK cells and similar levels to endogenous cells (Fig. 4d, right). This transfer system was also the ideal approach to address the involvement of NK cell-intrinsic TLR signaling in our endotoxemia model. Indeed, in vitro testing is hampered by the fact that a chronic lack of tonic TLR signaling lowers NK cell responsiveness in general, as indicated in literature(30). By pre-transferring TLR2/4KO NK cells, we found that both primary and memory responses of NK cells during LPS challenge were independent of TLR2 and 4 (fig S4c). Altogether, these results demonstrate that NK cells present at the moment of systemic inflammation, or descendants of the original cells, acquire memory properties after endotoxemia, in a TLR2 and -4-independent manner.

To determine whether the endotoxemia-induced memory cells have proliferated during the course of 14 days, we pre-transferred CFSE-labeled CD45.1 NK cells into CD45.2 mice followed by PBS/LPS injection (Fig. 4e). At day 14, in D14PBS mice we observed minimal CFSE dilution of pre-transferred NK cells (Fig. 4f). In contrast, in D14LPS mice, the majority of pre-transferred NK had divided as indicated by several peaks of decreasing CFSE signal (Fig. 4f,g). Our results thus demonstrate that D14LPS memory cells derive from NK cells that were exposed to systemic inflammation and maintain memory properties after undergoing several rounds of proliferation.

\section{Histone methylation at an ifng enhacer is involved in NK cell memory}

Since innate immune memory in macrophages and NK cells has been proposed to rely on epigenetic modifications, we explored whether this might be the case for post-endotoxemia memory NK cells. To investigate the possible epigenetic mechanism responsible from memory features we studied the histone marks associated with upstream regulatory regions of the ifng locus by chromatin immunoprecipitation followed by qPCR (ChIP-qPCR). We probed the ifng promoter and several possible enhancer regions, which had been previously described as being crucial for IFN $\gamma$ transcription(31). As positive and negative controls, we probed ActB, a housekeeping gene known to be expressed and Hoxc8, that is silent in hematopoietic cells, respectively (Fig. 5a). We focused on H3K4me1, a histone modification that marks active enhancers. Interestingly, we identified one region of the ifng locus, located $-22 \mathrm{~kb}$ 
from the transcriptional start site, that showed significant enrichment for H3K4me1 in D14LPS NK cells (Fig. 5b). The same region was not marked with H3K4me1 in D14PBS NK cells. Therefore, endotoxemia reveals a latent enhancer at the ifng locus, thereby poising NK cells for increased IFN $\gamma$ expression. Such results strongly suggest that post-endotoxemia NK cell memory has an epigenetic component.

To test the involvement of histone methylation on memory acquisition by NK cells after endotoxemia, we used a chemical methyltransferase inhibitor, sinefungin, previously reported to reduce H3K4me1 in vivo(32). We treated mice with sinefungin or saline, as a vehicle control, 2 days after PBS or LPS injection. At day 14 we purified NK cells from the 4 groups of mice (D14PBS - Saline, D14PBS Sinefungin, D14LPS - Saline and D14LPS - Sinefungin) and stimulated them with IL-15+IL-18+LPS in co-culture with congenic naïve NK cells. Strikingly, while saline treated D14LPS NK cells showed memory level responses, with higher IFN $\gamma+$ percentages, as compared to the co-cultured naïve NK cells and D14PBS NK cells, sinefungin treated D14LPS NK cells lost memory properties, responding at similar levels to co-cultured naïve NK cells (Fig. 5c). Importantly, sinefungin treatment did not block the response of D14PBS or D14LPS NK cells to IL-12+IL-18 (Fig. 5d). These results, demonstrate that methyltransferase-dependent modifications in D14LPS NKs are indeed acquired de novo, and influence only the memory responses (IL-15+IL-18+LPS) and not the naive response to cytokines IL-12+IL-18 of D14LPS NK cells. We further probed the ifng locus for H3K4me1 after sinefungin treatment. We perfomed ChIP-qPCR on NK cells from the respective groups of mice and found that sinefungin treatment blocked H3K4 methylation at the -22kb enhancer in D14LPS - Sinefungin mice (Fig. 5e), the condition in which memory response was abrogated. Therefore, NK cell memory to endotoxemia is mediated through a histone methylation-dependent mechanism, which reveals at least one latent enhancer at the ifng locus.

\section{Memory NK cells contribute to protection against bacterial infection}

To investigate the potential role of endotoxemia-induced memory NK cells in the context of bacterial infection we transferred approximately $1 \times 10^{5}$ highly purified D14PBS or D14LPS NK into naïve mice 
and infected them intraperitoneally with luminescent E. coli, 2 days after transfer. Bacteria were easily detected in the peritoneal cavity 15 minutes after infection (D0 in Fig. 6a) and could be followed for the next days. Noticeably, surviving mice, in which memory D14LPS NK cells were transfer, had a significantly reduced luminescent signal at day 1 post-infection compared to mice that received D14PBS NK cells (Fig. 6a,b). Memory NK cell transfer also limited mortality in recipient mice, with a 53.8\% survival rate as compared to only $25 \%$ in recipients of control NK cells (Fig. 6c). Therefore, these results suggest that post-endotoxemia memory NK cells play a beneficial role in bacterial infection by contributing to a reduction in bacterial burden.

\section{DISCUSSION}

In this study we show that LPS-induced systemic inflammation drives the formation of memory NK cells that persist for at least 9 weeks after endotoxemia, even under the control of a suppressive environment in the spleen. We demonstrate that NK memory is cell-intrinsic and is acquired by cells present at the time of systemic inflammation that undergo proliferation. We further provide molecular mechanism by which the memory is induced and/or maintained. We find that endotoxemia reveals a latent enhancer upstream of the ifng locus in NK cells, as seen by a dramatic increase in H3K4me1 levels, and that blocking methyltransferase activity can prevent acquisition of memory. Finally, we show that endotoxemia-induced memory NK cells can protect against bacterial infection.

Our findings that NK cells acquire memory properties, leading to enhanced activation upon restimulation after systemic inflammation, are surprising, since the consensus in literature is that NK cell functions are impaired during, and briefly following, acute episodes of sepsis(13). Indeed, when stimulated with cytokines, NK cells from acute phase sepsis patients or cecal-ligation and puncture (CLP) mice were reported to have suppressed IFN $\gamma$ production(16-18). NK cell cytotoxicity was also shown to be impaired $(19,33)$. However, very few studies have investigated the post-acute long-term effects of systemic inflammation on NK cells and their cell intrinsic activities independent of the immune environment. A recent report suggested that NK cell responsiveness was intact 7 days after bacterial and viral pneumonia, but their activation during secondary infection was hampered by the 
immunosuppressive environment in the lungs(34). These results are similar to what we present here, where 2-9 weeks after endotoxemia, the environment restricts NK cell activation during re-stimulation, independently of their cell-intrinsic functions. Previous reports have investigated the long-term immune consequences (up to 12 weeks) of systemic inflammation in CLP mice and showed that a suppressive environment is in place, including increased Treg frequencies and their suppressive capacity $(25,35$, 36), DC functional impairment(24) and expansion of immature myeloid cells(26). Similarly, we also observed increased frequencies of Tregs and expansion of myeloid cells, suggesting that endotoxemia induces similar long-lasting consequences to CLP. Thus, our results provide new evidence on the persistent effect of systemic inflammation on NK cell status. We show that while being within this suppressive environment, NK cell responsiveness to cytokine stimulation is not impaired. Most importantly, NK cell responsiveness to a second challenge with LPS is increased, in a cell intrinsic manner, providing the first evidence of NK cell memory following systemic inflammation.

Because of the nature of the LPS model we used in this study, a natural question is whether TLR4 is involved in endotoxemia-induced NK cell memory. Although LPS is the main ligand for TLR4, the role of this receptor for NK cells remains controversial. While several studies attributed a role for TLRs in NK cell activation $(37,38)$, TLR4 was shown to be dispensable for cell-intrinsic NK activation during endotoxemia in vivo, where NK cell response was mostly dependent on cytokines including IFN $\beta$, IL2, Il-15, IL-18 and IL-12(39). In line with these results, we found that activation of NK cells is not dependent on TLR2 and -4 during both primary and secondary responses in vivo. These results, along with our in vitro and in vivo data, where naïve and memory cells were subjected to the same inflammatory environment, argue for a minor role of cytokines in the increased responsiveness of memory NK cells. We believe that activation of NK cells during primary and secondary responses in our system would have to involve one or more NK cell receptors. Indeed, reports on NK cell memory to viruses and haptens show that specific receptors are involved in conferring a memory response(5). While suggested from the very first description of memory NK cells $(40)$, the role of the Ly49C/I receptors in establishing NK cell memory to hapten sensitization was just recently proven(41). Ly49C/I - MHC-I interactions were suggested to contribute to formation of memory responses by recognizing specific amino acid sequences on peptides. Similarly, human NK cells in the context of CMV infection 
also recognize specific $\mathrm{MCH}-\mathrm{I}$ presented peptides via the $\mathrm{NKG} 2 \mathrm{C}$ receptor $(8)$. Recognition of the $\mathrm{m} 157$ glycoprotein of MCVM by Ly49H( 7,42$)$ and of haptens by Ly49C/I $(40,41)$ in mice, and CMV peptides by the NKG2C receptor in humans $(8)$, substantiate the specificity of NK cells in both primary and recall responses. Indeed, receptor driven NK cell memory was shown to be exquisitely specific and contained within a subpopulation of cells carrying a given receptor $(8,40,41)$. Thus, the majority of memory NK cells that have been described are characterized by a receptor, which defines memory subpopulations within the total pool. While we have excluded the role of $\mathrm{Ly} 49 \mathrm{H}$ and $\mathrm{NKG} 2 \mathrm{~A} / \mathrm{C} / \mathrm{E}$, and we have not identified a specific receptor-defined sub-population responsible for mediating post-endotoxemia memory, the possibility remains that such a population exists.

Beyond the receptors at play in NK cell memory, the molecular mechanisms involved are just beginning to be uncovered. In human and mouse memory-like NK cells, changes in DNA methylation at the IFNG/ifng locus and at various signaling molecule loci was shown to be correlated with differential transcriptional responses, suggesting that an epigenetic component could be involved(10, 43, 44). Recently, an extensive study of chromatin accessibility on MCMV-specific Ly49H memory NK cells using ATAC-Seq was performed(11). These results highlighted that memory NK possess distinct chromatin accessibility states, similarly to memory CD8 cells. However, how this regulates gene expression remains unknown. Here we show that memory NK cells acquire H3K4me1 at an upstream region of the ifng locus, previously identified to be crucial for IFN $\gamma$ expression and suggested to be an enhancer(31). Such, de novo marking of an enhancer was observed in memory CD8 T cells following LCMV infection(45), but has not been reported for NK cells. In addition, H3K4me1 enhancer marking in macrophages is retained upon signal termination and is important for the augmented response observed upon re-stimulation(46). Interestingly, such data along with ours, strongly suggest that the enhancer repertoire of a cell is not fixed upon terminal differentiation, can be expanded by external stimuli, and can be maintained for a memory response.

NK cell memory is well described for hapten-sensitization, viral infection and in vitro cytokine treatment(5), however, evidence of NK cell memory to bacterial infection or sepsis is scarce. One report described a mouse model of BCG vaccination and Mycobacterium tuberculosis infection showing an 
expansion of IFNg+NKp46+CD27+ cells during re-infection and that transfer of these cells into naïve mice could help control bacterial burden(47). The authors also observed similar expansion of memorylike cells upon in vitro stimulation of peripheral blood mononuclear cells from humans with latent TB. NK cells from these samples could control macrophage infection with M. tuberculosis in vitro. We find that systemic inflammation induced by LPS also elicits NK cell memory and these memory NK cells can protect against $E$. coli infection when transferred into naïve mice. These studies and ours imply that NK memory to bacteria is relevant, and therefore memory NK cells could be considered as vaccination targets and efficacy read-outs. This prospect is especially interesting given the important role NK cells play during bacterial infection and their potency in treating such diseases as cancer $(15,48)$. In addition, our finding that NK cells responsiveness to bacterial products is heightened, while that of the environment is suppressed, suggests that NK cells could be good targets for immunotherapy in order to modulate the post-sepsis immune system. 


\section{MATHERIALS AND METHODS}

\section{Mice and endotoxemia model}

All protocols for animal experiments were reviewed and approved by the CETEA (Comité d'Ethique pour l'Expérimentation Animale - Ethics Committee for Animal Experimentation) of the Institut Pasteur under approval number 2016-003 and were performed in accordance with national laws and institutional guidelines for animal care and use. CD45.1, CD45.2 and CD45.1/2 mice, male and females of 8 to 12 weeks were purchased from Janvier (France) or breed at the Institut Pasteur animal facility. For endotoxemia, mice were injected intraperitoneally with a single dose of conventional LPS from E. coli O111:B4 (Sigma Aldrich), $10 \mathrm{mg} / \mathrm{kg}$ in a $200 \mu \mathrm{l}$ volume of PBS. Control mice received just PBS injections. Animals were monitored daily for the first 5 days and then weekly for the duration of the experiment. Animals were sacrificed in the event they lost more than $20 \%$ of weight as a result of endotoxemia (mortality was usually below 10\% at this dose of LPS). A clinical score was used to assess progression of mice throughout endotoxemia, representing the sum of the following scores: 0 - no clinical signs; 1 - hypoactivity; 1 - ruffled fur; 2 -hunched posture; 2 - diarrhoea; 3 - prostration. For histone methyltransferase inhibition experiments, mice were treat at day 2 after PBS/LPS injection with saline or Sinefungin (Abcam) $(10 \mathrm{mg} / \mathrm{kg}$, i.p. in a $200 \mu \mathrm{l}$ volume of saline.

\section{Cytometry staining protocols and analysis}

Single cell suspensions from spleens were counted and prepared for surface staining in 96 well plates. Unspecific binding was first blocked by incubation with anti-mouse CD16/CD32 (BD Biosciences) for 10 minutes before addition of surface labelling antibodies for another 45 minutes, in $0,5 \% \mathrm{FCS}$ at $4{ }^{\circ} \mathrm{C}$. Cells were washed twice in PBS in preparation for viability staining using fixable viability dye (eFluor780, ebioscience) for 5 minutes at $4^{\circ} \mathrm{C}$. Cell were washed twice in $0,5 \%$ FCS and fixed using commercial fixation buffer (Biolegend). For intracellular cytokine and Foxp3 staining, cells were permeabilized and washed with buffers from commercial kits (Inside Stain Kit, Miltenyi Biotec and Foxp3 / Transcription Factor Fixation/Permeabilization Concentrate and Diluent, eBioscience, respectively). Following permeabilization and washing, cells were stained with respective antibodies in $0,5 \% \mathrm{FCS}$ for 30 minutes at $4^{\circ} \mathrm{C}$ and after a final washing suspended in buffer for FACS analysis. Sample 
acquisition was performed on a MACSQuant flow-cytometer (Miltenyi Biotec) and analysis was done using FlowJo Software (TreeStar).

\section{Antibodies}

The following antibodies (clones) were used: NK1.1 (PK136), CD3 (145-2C11), CD49b (DX5), CD69 (H1.2F3), Ly6C (HK1.4), Ly6G (1A8-Ly6g), CD11b (M1/70), CD11c (HL3), Ly49H (REA241), Ly49F (HBF-719), Ly49D (4E5), Ly49C/I (REA253), NKG2A/C/E (20d5), NKG2D (CX5), CD45.1 (A20), CD45.2 (104), foxp3 (FJK-16s) and IFN $\gamma$ (XMG1.2) were purchased from Biolegend, eBioscience, BD Biosciences or Miltenyi Biotec.

\section{NK cell enrichment and purification}

Splenocytes obtained by mechanical dissociation of spleens were passed successively through $100 \mu \mathrm{m}$, $70 \mu \mathrm{m}$ and $30 \mu \mathrm{m}$ strainers (Milteny Biotec) and counted before use for downstream applications. NK cells were enriched from splenocyte suspensions using negative enrichment kits (eBioscience) according to manufacturer's protocol but combined with separation over magnetic columns (LS columns, Miltenyi Biotec) and were routinely brought to $80 \%$ purity. For some in vitro stimulations and transfers of cells before bacterial challenge, enriched NK cells were re-purified using mouse NK cell purification kits (Miltenyi Biotec) according to manufacturer's instructions to reach purities of approximately $98 \%$.

\section{Cell transfer experiments}

Enriched NK cells from CD45.1, CD45.2 or CD45.1/2 mice were transferred i.v. into recipient congenic mice $\left(0,75-1 \times 10^{6}\right.$ cells/recipient) in $100 \mu 1$ of PBS. For forward transfers, NK cells were transferred into congenic naïve mice 1 day before LPS challenge. At 6 hours after PBS or LPS injection, single cell suspensions were obtained from spleen of recipient mice and flow cytometric analysis was performed. For pre-/post-transfer experiments, CD45.1 NK cells were pre-transferred into CD45.1/2 mice 1 day before endotoxemia induction and naïve CD45.2 NK cells were post-transferred 7 days after. At day 14 after endotoxemia, mice were re-challenged with PBS/LPS and NK cell responses were assessed by flow cytometry as described above. For proliferation assessment experiments, enriched NK cells were stained with CFSE $2,5 \mu \mathrm{M}$ in PBS for 10 minutes, before blocking in FCS and extensively washed with 
PBS before transfer into congenic mice, 1 day before endotoxemia induction. For bacterial challenge experiments $10^{5}$ highly-purified NK cells from D13PBS or LPS mice were transferred to naïve 2 days before challenge with $2-4 \times 10^{7}$ E. coli intraperitoneally.

\section{NK cell culture and co-cultures}

Purified NK cells were cultured at $1 \times 10^{6}$ cells $/ \mathrm{ml}$ in $200 \mu 1$ RPMI 1640 (Gibco) supplemented with 10\% FCS and Penicillin-Streptomycin (Gibco) respectively in 96 well round-bottom plates (Nunc). Cells were either left unstimulated or activated with conventional LPS from E. coli O111:B4 (Sigma Aldrich) at $100 \mathrm{ng} / \mathrm{ml}$ or various cytokine cocktails composed of IL-15, IL-12 (Miltenyi Biotec) and IL-18 (MBL International) at a dose of $10 \mathrm{ng} / \mathrm{ml}$ each. NK cell were additionally stimulated with LPS + cytokine cocktails. After 20 hours of culture, cells were collected for intracellular cytokine staining by flow cytometry and culture supernatants were collected and stored at $-20^{\circ} \mathrm{C}$ for cytokine assays performed by ELISA according to manufacturer's instructions (DuoSet, R\&D Systems).

\section{ChIP-qPCR assays}

ChIP was performed as described before by Batsche et al(49) with some modifications. NK cells were fixed in $1 \%$ formaldehyde ( $8 \mathrm{~min}$, room temperature), and the reaction was stopped by the addition of glycine at the final concentration of $0,125 \mathrm{M}$. After two washes in PBS, cells were resuspended in $0.25 \%$ Triton X-100, $10 \mathrm{mM}$ Tris-HCl (pH 8), $10 \mathrm{mM}$ EDTA, $0.5 \mathrm{mM}$ EGTA and proteases inhibitors; the soluble fraction was eliminated by centrifugation; and chromatin was extracted with $250 \mathrm{mM} \mathrm{NaCl}, 50$ mM Tris- $\mathrm{HCl}(\mathrm{pH} 8), 1 \mathrm{mM}$ EDTA, $0.5 \mathrm{mM}$ EGTA and proteases inhibitors cocktail for $30 \mathrm{~min}$ on ice. Chromatin was resuspended in 1\% SDS, $10 \mathrm{mM}$ Tris-HCl (pH 8), 1 mM EDTA, 0.5 mM EGTA and proteases inhibitors cocktail; and sonicated during 6 cycles using Diagenode Bioruptor Pico (15 sec on, $30 \mathrm{sec}$ off). DNA fragment size $(<1 \mathrm{~kb})$ was verified by agarose gel electrophoresis. ChIP was performed using $\mathrm{H} 3$, H3K4me3, H3K4me1 antibodies and nonimmune IgG (negative control antibody), chromatin extracted from $3 \times 10^{6} \mathrm{NK}$ cells per condition was used. Chromatin was diluted 10 times in $0.6 \%$ Triton $\mathrm{X}-100,0.06 \%$ sodium deoxycholate (NaDOC), $150 \mathrm{mM} \mathrm{NaCl}, 12 \mathrm{mM}$ Tris-HCl, $1 \mathrm{mM}$ EDTA, $0.5 \mathrm{mM}$ EGTA and proteases inhibitors cocktail. For 6 hours, the different antibodies were previously incubated at $4{ }^{\circ} \mathrm{C}$ with protein G-coated magnetic beads (DiaMag, Diagenode), protease inhibitor cocktail and $0.1 \%$ BSA. Chromatin was incubated overnight at $4^{\circ} \mathrm{C}$ with each antibody/protein G-coated magnetic beads. 
Immunocomplexes were washed with $1 \mathrm{x}$ buffer 1 (1\% Triton X-100, 0.1\% NaDOC, $150 \mathrm{mM} \mathrm{NaCl}, 10$ $\mathrm{mM}$ Tris- $\mathrm{HCl}(\mathrm{pH})), 1 \mathrm{x}$ buffer $2(0.5 \%$ NP-40, 0.5\% Triton X-100, $0.5 \mathrm{NaDOC}, 150 \mathrm{mM} \mathrm{NaCl}, 10$ $\mathrm{mM}$ Tris- $\mathrm{HCl}(\mathrm{pH} 8)), 1 \times$ buffer $3(0.7 \%$ Triton X-100, 0.1\% NaDOC, $250 \mathrm{mM} \mathrm{NaCl}, 10 \mathrm{mM}$ Tris$\mathrm{HCl}(\mathrm{pH} 8)) 1 \mathrm{x}$ buffer $4(0.5 \%$ NP-40, 0.5\% NaDOC, $250 \mathrm{mM} \mathrm{LiCl}, 20 \mathrm{mM}$ Tris-HCl (pH 8), $1 \mathrm{mM}$ EDTA), 1 x buffer $5(0,1 \%$ NP-40, $150 \mathrm{mM} \mathrm{NaCl}, 20 \mathrm{mM}$ Tris-HCl (pH 8), $1 \mathrm{mM}$ EDTA) and $1 \mathrm{x}$ buffer 6 (20mM Tris-HCl (pH 8), 1 mM EDTA) . Beads were eluted in water containing $10 \%$ Chelex and reverse cross-linked by boiling for $10 \mathrm{~min}$, incubating with RNase for $10 \mathrm{~min}$ at room temperature, then with proteinase $\mathrm{K}$ for $20 \mathrm{~min}$ at $55^{\circ} \mathrm{C}$ and reboiling for $10 \mathrm{~min}$. DNA fragment were purified by Phenol Chloroform extraction.

Amplifications (40 cycles) were performed using quantitative real-time PCR using iTaq ${ }^{\mathrm{TM}}$ Universal Syber Green Supermix (BIORAD) on a CFX384 Touch Real-Time PCR system (BIORAD). qPCR efficiency (E) was determined for the ChIP primers with a dilution series of genomic mouse DNA. The threshold cycles (Ct values) were recordered from the exponential phase of the qPCR for IP and input DNA for each primer pair. The relative amont of immunoprecipitated DNA was comparated to input DNA for the control regions ( $\%$ of recovery) using the following formula: $\%$ recovery $=\mathrm{E}^{\wedge}((\mathrm{Ct}(1 \%$ input) - $\log _{2}($ input dilution) $\left.)-C t(I P)\right) \times 100$. Antibodies ChIP-grade and ChIP primers sequences are provided in Supplemental Table 1

\section{Statistical analysis}

Statistical significance was tested using Prism 5.0 Software (GraphPad). Mann Whitney test was used for single comparisons and Wilcoxon paired test for paired comparison of samples in the same mouse in transfer experiments or in the same well for co-culture experiments. Unless otherwise specified in figure legends, error bars in all figures represent SEM, with the midlines representing the mean value. 


\section{ACKNOWLEDGEMENTS}

Work in the M.A.H. laboratory received financial support from Institut Pasteur and the National Research Agency (ANR-EPIBACTIN). O.R. was supported by a stipend from the Pasteur - Paris University International Ph.D. Program for part of this work. T.C. is supported by a fellowship from the Foundation for Medical Research (Marianne Josso Prize).

\section{COMPETING INTERESTS}

The authors declare no competing interests.

\section{CONTRIBUTIONS}

O.R. coordinated the study; O.R., J-M.C. and M.A.H. conceived of and designed the study; O.R., T.C., C.C. and C.F. performed experiments and analyzed data; O.R. and M.A.H. conceived and wrote the manuscript; J-M.C. edited the manuscript; J-M.C. and M.A.H. supervised the work 


\section{REFERENCES}

1. J. C. Sun, S. Ugolini, E. Vivier, Immunological memory within the innate immune system. EMBO J 33, 1295-1303 (2014).

2. M. G. Netea, J. Quintin, J. W. van der Meer, Trained immunity: a memory for innate host defense. Cell Host Microbe 9, 355-361 (2011).

3. M. G. Netea, L. A. Joosten, E. Latz, K. H. Mills, G. Natoli, H. G. Stunnenberg, L. A. O'Neill, R. J. Xavier, Trained immunity: A program of innate immune memory in health and disease. Science 352, aaf1098 (2016).

4. S. Saeed, J. Quintin, H. H. Kerstens, N. A. Rao, A. Aghajanirefah, F. Matarese, S. C. Cheng, J. Ratter, K. Berentsen, M. A. van der Ent, N. Sharifi, E. M. Janssen-Megens, M. Ter Huurne, A. Mandoli, T. van Schaik, A. Ng, F. Burden, K. Downes, M. Frontini, V. Kumar, E. J. Giamarellos-Bourboulis, W. H. Ouwehand, J. W. van der Meer, L. A. Joosten, C. Wijmenga, J. H. Martens, R. J. Xavier, C. Logie, M. G. Netea, H. G. Stunnenberg, Epigenetic programming of monocyte-to-macrophage differentiation and trained innate immunity. Science 345, 1251086 (2014).

5. A. Cerwenka, L. L. Lanier, Natural killer cell memory in infection, inflammation and cancer. Nature reviews. Immunology 16, 112-123 (2016).

6. C. D. Geary, J. C. Sun, Memory responses of natural killer cells. Semin Immunol 31, 11-19 (2017).

7. J. C. Sun, J. N. Beilke, L. L. Lanier, Adaptive immune features of natural killer cells. Nature 457, 557-561 (2009).

8. Q. Hammer, T. Ruckert, E. M. Borst, J. Dunst, A. Haubner, P. Durek, F. Heinrich, G. Gasparoni, M. Babic, A. Tomic, G. Pietra, M. Nienen, I. W. Blau, J. Hofmann, I. K. Na, I. Prinz, C. Koenecke, P. Hemmati, N. Babel, R. Arnold, J. Walter, K. Thurley, M. F. Mashreghi, M. Messerle, C. Romagnani, Peptide-specific recognition of human cytomegalovirus strains controls adaptive natural killer cells. Nat Immunol 19, 453-463 (2018).

9. B. Tesi, H. Schlums, F. Cichocki, Y. T. Bryceson, Epigenetic Regulation of Adaptive NK Cell Diversification. Trends Immunol 37, 451-461 (2016).

10. M. Luetke-Eversloh, Q. Hammer, P. Durek, K. Nordstrom, G. Gasparoni, M. Pink, A. Hamann, J. Walter, H. D. Chang, J. Dong, C. Romagnani, Human cytomegalovirus drives epigenetic imprinting of the IFNG locus in NKG2Chi natural killer cells. PLoS Pathog 10, e1004441 (2014).

11. C. M. Lau, N. M. Adams, C. D. Geary, O. E. Weizman, M. Rapp, Y. Pritykin, C. S. Leslie, J. C. Sun, Epigenetic control of innate and adaptive immune memory. Nat Immunol 19, 963-972 (2018).

12. J. M. Cavaillon, M. Adib-Conquy, C. Fitting, C. Adrie, D. Payen, Cytokine cascade in sepsis. Scand J Infect Dis 35, 535-544 (2003).

13. Y. Guo, N. K. Patil, L. Luan, J. K. Bohannon, E. R. Sherwood, The biology of natural killer cells during sepsis. Immunology 153, 190-202 (2018).

14. L. Chiche, J. M. Forel, G. Thomas, C. Farnarier, F. Vely, M. Blery, L. Papazian, E. Vivier, The role of natural killer cells in sepsis. J Biomed Biotechnol 2011, 986491 (2011).

15. F. Souza-Fonseca-Guimaraes, M. Adib-Conquy, J. M. Cavaillon, Natural killer (NK) cells in antibacterial innate immunity: angels or devils? Molecular medicine 18, 270-285 (2012).

16. F. Souza-Fonseca-Guimaraes, M. Parlato, C. Fitting, J. M. Cavaillon, M. Adib-Conquy, NK cell tolerance to TLR agonists mediated by regulatory T cells after polymicrobial sepsis. Journal of immunology 188, 5850-5858 (2012).

17. F. Souza-Fonseca-Guimaraes, M. Parlato, F. Philippart, B. Misset, J. M. Cavaillon, M. AdibConquy, g. Captain study, Toll-like receptors expression and interferon-gamma production by NK cells in human sepsis. Crit Care 16, R206 (2012).

18. L. Chiche, J. M. Forel, G. Thomas, C. Farnarier, C. Cognet, C. Guervilly, C. Zandotti, F. Vely, A. Roch, E. Vivier, L. Papazian, Interferon-gamma production by natural killer cells and cytomegalovirus in critically ill patients. Crit Care Med 40, 3162-3169 (2012). 
19. L. von Muller, A. Klemm, N. Durmus, M. Weiss, H. Suger-Wiedeck, M. Schneider, W. Hampl, T. Mertens, Cellular immunity and active human cytomegalovirus infection in patients with septic shock. J Infect Dis 196, 1288-1295 (2007).

20. J. M. Cavaillon, E. J. Giamarellos-Bourboulis, $<<$ Immunosuppression $>>$ is Inappropriately Qualifying the Immune Status of Septic and Sirs Patients. Shock, (2018).

21. M. Shankar-Hari, G. D. Rubenfeld, Understanding Long-Term Outcomes Following Sepsis: Implications and Challenges. Curr Infect Dis Rep 18, 37 (2016).

22. E. Vivier, E. Tomasello, M. Baratin, T. Walzer, S. Ugolini, Functions of natural killer cells. Nat Immunol 9, 503-510 (2008).

23. O. Rasid, I. S. Ciulean, C. Fitting, N. Doyen, J. M. Cavaillon, Local Microenvironment Controls the Compartmentalization of NK Cell Responses during Systemic Inflammation in Mice. Journal of immunology 197, 2444-2454 (2016).

24. C. F. Benjamim, S. K. Lundy, N. W. Lukacs, C. M. Hogaboam, S. L. Kunkel, Reversal of longterm sepsis-induced immunosuppression by dendritic cells. Blood 105, 3588-3595 (2005).

25. K. A. Cavassani, W. F. t. Carson, A. P. Moreira, H. Wen, M. A. Schaller, M. Ishii, D. M. Lindell, Y. Dou, N. W. Lukacs, V. G. Keshamouni, C. M. Hogaboam, S. L. Kunkel, The post sepsisinduced expansion and enhanced function of regulatory $\mathrm{T}$ cells create an environment to potentiate tumor growth. Blood 115, 4403-4411 (2010).

26. M. J. Delano, P. O. Scumpia, J. S. Weinstein, D. Coco, S. Nagaraj, K. M. Kelly-Scumpia, K. A. O'Malley, J. L. Wynn, S. Antonenko, S. Z. Al-Quran, R. Swan, C. S. Chung, M. A. Atkinson, R. Ramphal, D. I. Gabrilovich, W. H. Reeves, A. Ayala, J. Phillips, D. Laface, P. G. Heyworth, M. Clare-Salzler, L. L. Moldawer, MyD88-dependent expansion of an immature GR$1(+) \mathrm{CD} 11 \mathrm{~b}(+)$ population induces $\mathrm{T}$ cell suppression and Th2 polarization in sepsis. The Journal of experimental medicine 204, 1463-1474 (2007).

27. G. Min-Oo, L. L. Lanier, Cytomegalovirus generates long-lived antigen-specific NK cells with diminished bystander activation to heterologous infection. The Journal of experimental medicine 211, 2669-2680 (2014).

28. S. Lopez-Verges, J. M. Milush, B. S. Schwartz, M. J. Pando, J. Jarjoura, V. A. York, J. P. Houchins, S. Miller, S. M. Kang, P. J. Norris, D. F. Nixon, L. L. Lanier, Expansion of a unique CD57(+)NKG2Chi natural killer cell subset during acute human cytomegalovirus infection. Proc Natl Acad Sci U S A 108, 14725-14732 (2011).

29. Y. Gao, F. Souza-Fonseca-Guimaraes, T. Bald, S. S. Ng, A. Young, S. F. Ngiow, J. Rautela, J. Straube, N. Waddell, S. J. Blake, J. Yan, L. Bartholin, J. S. Lee, E. Vivier, K. Takeda, M. Messaoudene, L. Zitvogel, M. W. L. Teng, G. T. Belz, C. R. Engwerda, N. D. Huntington, K. Nakamura, M. Holzel, M. J. Smyth, Tumor immunoevasion by the conversion of effector NK cells into type 1 innate lymphoid cells. Nat Immunol 18, 1004-1015 (2017).

30. S. C. Ganal, S. L. Sanos, C. Kallfass, K. Oberle, C. Johner, C. Kirschning, S. Lienenklaus, S. Weiss, P. Staeheli, P. Aichele, A. Diefenbach, Priming of natural killer cells by nonmucosal mononuclear phagocytes requires instructive signals from commensal microbiota. Immunity $\mathbf{3 7}$, 171-186 (2012).

31. R. D. Hatton, L. E. Harrington, R. J. Luther, T. Wakefield, K. M. Janowski, J. R. Oliver, R. L. Lallone, K. M. Murphy, C. T. Weaver, A distal conserved sequence element controls Ifng gene expression by T cells and NK cells. Immunity 25, 717-729 (2006).

32. K. Sasaki, S. Doi, A. Nakashima, T. Irifuku, K. Yamada, K. Kokoroishi, T. Ueno, T. Doi, E. Hida, K. Arihiro, N. Kohno, T. Masaki, Inhibition of SET Domain-Containing Lysine Methyltransferase 7/9 Ameliorates Renal Fibrosis. J Am Soc Nephrol 27, 203-215 (2016).

33. I. J. Jensen, C. S. Winborn, M. G. Fosdick, P. Shao, M. M. Tremblay, Q. Shan, S. K. Tripathy, C. M. Snyder, H. H. Xue, T. S. Griffith, J. C. Houtman, V. P. Badovinac, Polymicrobial sepsis influences NK-cell-mediated immunity by diminishing NK-cell-intrinsic receptor-mediated effector responses to viral ligands or infections. PLoS Pathog 14, e1007405 (2018).

34. A. Roquilly, H. E. G. McWilliam, C. Jacqueline, Z. Tian, R. Cinotti, M. Rimbert, L. Wakim, I. Caminschi, M. H. Lahoud, G. T. Belz, A. Kallies, J. D. Mintern, K. Asehnoune, J. A. Villadangos, Local Modulation of Antigen-Presenting Cell Development after Resolution of Pneumonia Induces Long-Term Susceptibility to Secondary Infections. Immunity 47, 135-147 e135 (2017). 
35. H. Wen, Y. Dou, C. M. Hogaboam, S. L. Kunkel, Epigenetic regulation of dendritic cell-derived interleukin-12 facilitates immunosuppression after a severe innate immune response. Blood 111, 1797-1804 (2008).

36. D. C. Nascimento, P. H. Melo, A. R. Pineros, R. G. Ferreira, D. F. Colon, P. B. Donate, F. V. Castanheira, A. Gozzi, P. G. Czaikoski, W. Niedbala, M. C. Borges, D. S. Zamboni, F. Y. Liew, F. Q. Cunha, J. C. Alves-Filho, IL-33 contributes to sepsis-induced long-term immunosuppression by expanding the regulatory T cell population. Nat Commun 8, 14919 (2017).

37. J. Martinez, X. Huang, Y. Yang, Direct TLR2 signaling is critical for NK cell activation and function in response to vaccinia viral infection. PLoS Pathog 6, e1000811 (2010).

38. A. Chalifour, P. Jeannin, J. F. Gauchat, A. Blaecke, M. Malissard, T. N'Guyen, N. Thieblemont, Y. Delneste, Direct bacterial protein PAMP recognition by human NK cells involves TLRs and triggers alpha-defensin production. Blood 104, 1778-1783 (2004).

39. I. Zanoni, R. Spreafico, C. Bodio, M. Di Gioia, C. Cigni, A. Broggi, T. Gorletta, M. Caccia, G. Chirico, L. Sironi, M. Collini, M. P. Colombo, N. Garbi, F. Granucci, IL-15 cis presentation is required for optimal NK cell activation in lipopolysaccharide-mediated inflammatory conditions. Cell Rep 4, 1235-1249 (2013).

40. J. G. O'Leary, M. Goodarzi, D. L. Drayton, U. H. von Andrian, T cell- and B cell-independent adaptive immunity mediated by natural killer cells. Nat Immunol 7, 507-516 (2006).

41. A. Wight, A. B. Mahmoud, M. Scur, M. M. Tu, M. M. A. Rahim, S. Sad, A. P. Makrigiannis, Critical role for the Ly49 family of class I MHC receptors in adaptive natural killer cell responses. Proc Natl Acad Sci U S A 115, 11579-11584 (2018).

42. H. R. Smith, J. W. Heusel, I. K. Mehta, S. Kim, B. G. Dorner, O. V. Naidenko, K. Iizuka, H. Furukawa, D. L. Beckman, J. T. Pingel, A. A. Scalzo, D. H. Fremont, W. M. Yokoyama, Recognition of a virus-encoded ligand by a natural killer cell activation receptor. Proc Natl Acad Sci U S A 99, 8826-8831 (2002).

43. H. Schlums, F. Cichocki, B. Tesi, J. Theorell, V. Beziat, T. D. Holmes, H. Han, S. C. Chiang, B. Foley, K. Mattsson, S. Larsson, M. Schaffer, K. J. Malmberg, H. G. Ljunggren, J. S. Miller, Y. T. Bryceson, Cytomegalovirus infection drives adaptive epigenetic diversification of NK cells with altered signaling and effector function. Immunity 42, 443-456 (2015).

44. J. Lee, T. Zhang, I. Hwang, A. Kim, L. Nitschke, M. Kim, J. M. Scott, Y. Kamimura, L. L. Lanier, S. Kim, Epigenetic modification and antibody-dependent expansion of memory-like NK cells in human cytomegalovirus-infected individuals. Immunity 42, 431-442 (2015).

45. B. He, S. Xing, C. Chen, P. Gao, L. Teng, Q. Shan, J. A. Gullicksrud, M. D. Martin, S. Yu, J. T. Harty, V. P. Badovinac, K. Tan, H. H. Xue, CD8(+) T Cells Utilize Highly Dynamic Enhancer Repertoires and Regulatory Circuitry in Response to Infections. Immunity 45, 13411354 (2016).

46. R. Ostuni, V. Piccolo, I. Barozzi, S. Polletti, A. Termanini, S. Bonifacio, A. Curina, E. Prosperini, S. Ghisletti, G. Natoli, Latent enhancers activated by stimulation in differentiated cells. Cell 152, 157-171 (2013).

47. S. Venkatasubramanian, S. Cheekatla, P. Paidipally, D. Tripathi, E. Welch, A. R. Tvinnereim, R. Nurieva, R. Vankayalapati, IL-21-dependent expansion of memory-like NK cells enhances protective immune responses against Mycobacterium tuberculosis. Mucosal Immunol 10, 10311042 (2017).

48. A. Horowitz, K. A. Stegmann, E. M. Riley, Activation of natural killer cells during microbial infections. Front Immunol 2, 88 (2011).

49. E. Batsche, M. Yaniv, C. Muchardt, The human SWI/SNF subunit Brm is a regulator of alternative splicing. Nat Struct Mol Biol 13, 22-29 (2006). 


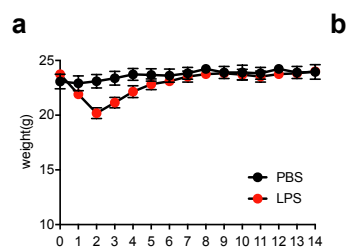

b

d
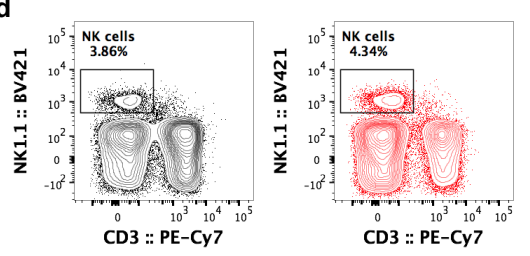

Total spleen cell stimulation
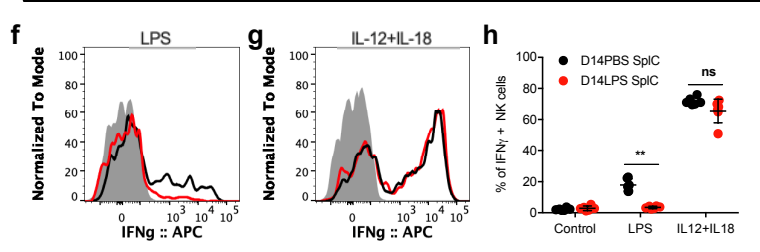

NK cell stimulation
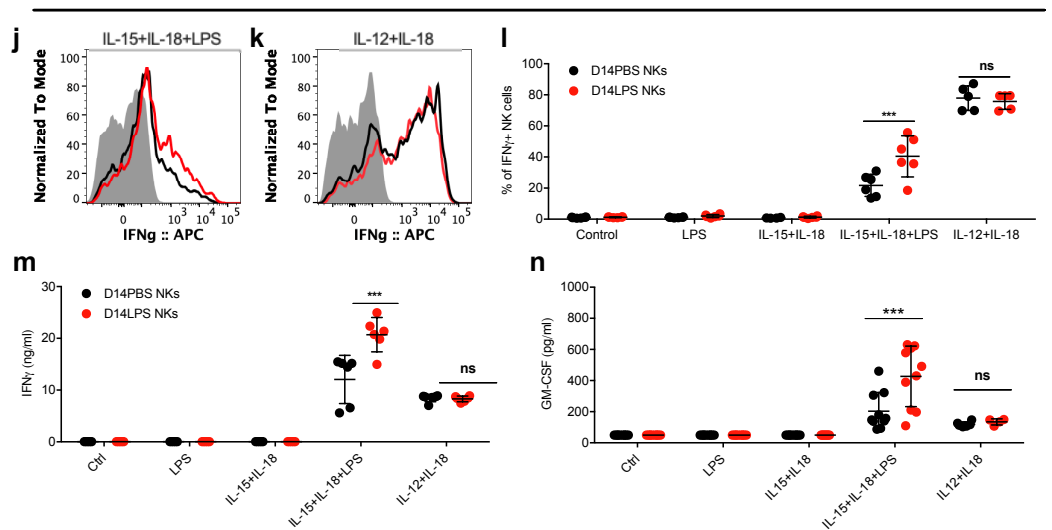

n

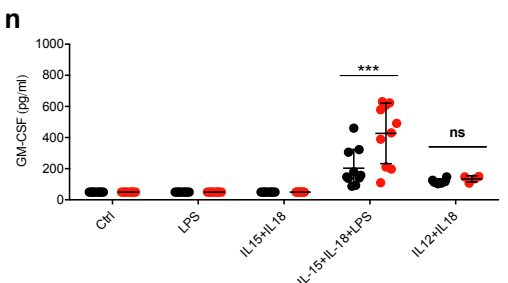

Fig. 1 NK cells acquire memory-like features 14 days after endotoxemia

C57BL/6 mice were injected with either PBS or LPS $(10 \mathrm{mg} / \mathrm{kg})$ intraperitoneally and monitored 14 after which they were used to assess NK cell status ex vivo. a) Weight, b) Clinical score and c) Survival monitoring following injection of PBS or LPS. Spleen NK cells from D14PBS and D14LPS mice were analyzed: d) Representative gating for NK cells in both conditions e) Percentages of NK cells among lymphocytes and total NK cell numbers. Splenocytes (SplC) from D14PBS and LPS mice were stimulated in vitro with cytokines $(10 \mathrm{ng} / \mathrm{ml})$ or LPS $(100 \mathrm{ng} / \mathrm{ml})$ overnight. Cell were stained for flow cytometry analysis to assess levels of intracellular IFN $\gamma$ and supernatants were collected for cytokine ELISAs. f-g) Representative overlay histograms and $\mathbf{h}$ ) quantification of IFN $\gamma$ expression by NK cells in respective conditions. i) Total IFN $\gamma$ levels in supernatants from SplC. NK cells were enriched from spleens of D14PBS and LPS mice and stimulated in vitro with cytokines and/or LPS overnight. $\mathbf{j}$-k) Representative overlay histograms and l) quantification of IFN $\gamma$ expression by NK cells in respective conditions. Total $\mathbf{m}$ ) IFN $\gamma$ and $\mathbf{n}$ ) GM-CSF levels in supernatants from NK cells in respective conditions. Dots represent individual mice. Data represent one out of least 3 repeats with $\mathrm{n} \geq 5$ mice/group. ns, not significant, ${ }^{*} \mathrm{p}<0.05,{ }^{*} \mathrm{p}<0.01,{ }^{* * *} \mathrm{p}<0.001$. Mann-Whitney test comparing D14PBS and D14LPS cell values. 

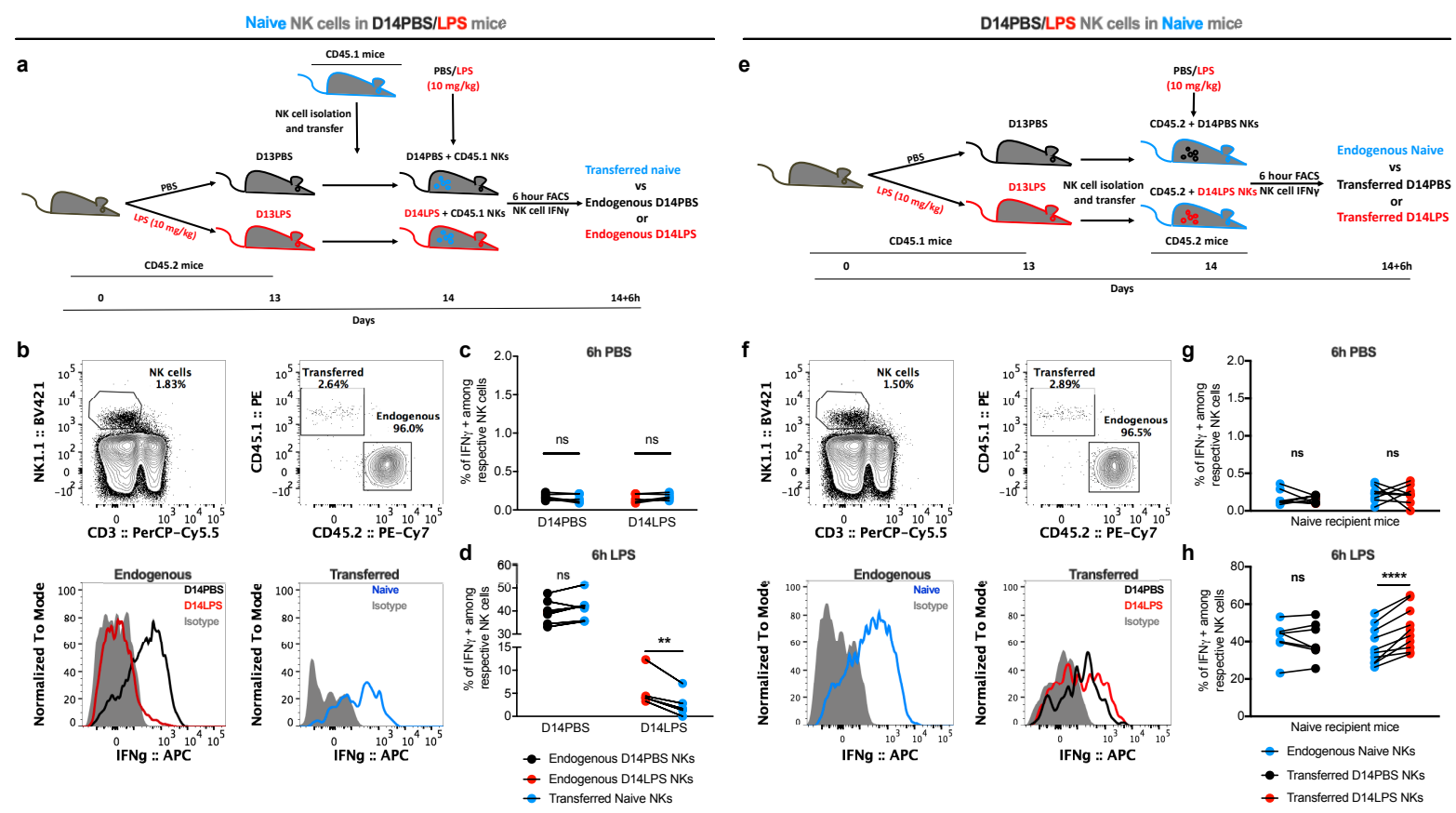

Fig. 2 NK cells retain cell-intrinsic memory properties in vivo after endotoxemia under a suppressive environment.

NK cells enriched from spleens of naïve CD45.1 mice were transferred into CD45.2 mice $\left(1 \times 10^{6}\right.$ cells/recipient, intravenously) injected 13 days before with PBS (D13PBS) or LPS (10mg/kg, i.p.) (D13LPS). Recipient mice were challenged one day later with PBS or LPS $(10 \mathrm{mg} / \mathrm{kg})$ and sacrificed 6 hours later. Splenocytes were processed for flow cytometry to levels of intracellular IFN $\gamma$ in endogenous vs transferred NK cells. a) Experimental transfer scheme b) Representative gating strategy c) Data summary grouped before-after plots depicting percentages of IFN $\gamma+$ cells among endogenous CD45.2 D14PBS NK cells (black dots), transferred CD45.1 naive NK cells (blue dots) and endogenous CD45.2 D14LPS NK cells (red dots) at 6 hours after PBS or d) LPS rechallenge. NK cells were enriched from spleens of CD45.1 mice injected 13 days before with PBS (D13PBS) or LPS (10mg/kg, i.p.) (D13LPS) and were transferred into naïve CD45.2 mice ( $1 \times 10^{6}$ cells/recipient, intravenously). Recipient mice were challenged and samples harvested as above. e) Experimental transfer scheme f) Representative gating strategy g) Data summary grouped before-after plots depicting percentages of IFN $\gamma+$ cells among endogenous CD45.2 naive NK cells (blue dots), transferred D14PBS CD45.1 NK cells (black dots) and transferred D14LPS CD45.2 NK cells (red dots) at 6 hours after PBS or h) LPS re-challenge. Dots represent individual mice. Data are representative of one experiment out of 3 repeats with $n \geq 5$ mice/group. ns, not significant, ${ }^{*} \mathrm{p}<0.05$. Wilcoxon paired test comparing D14Sln, D14LPS and naïve cell values under respective conditions. 
a

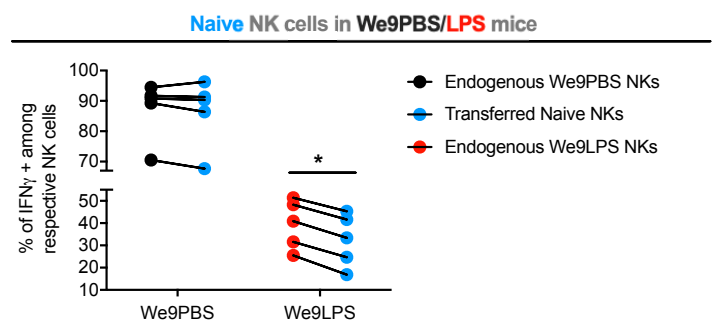

c

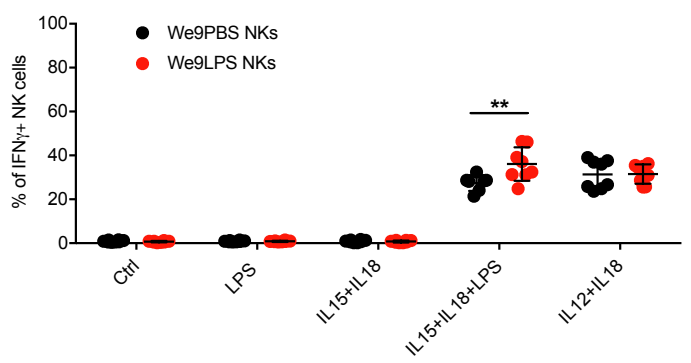

b

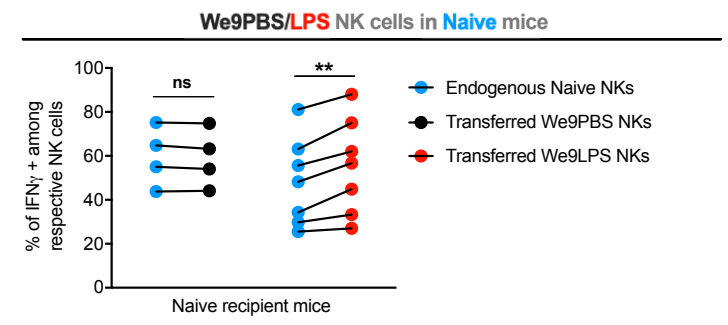

d

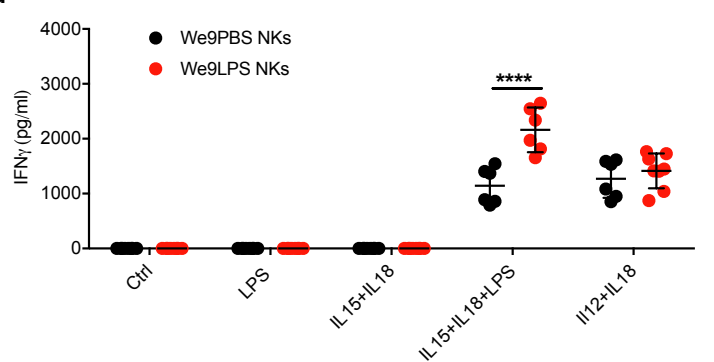

Fig. 3 NK cells retain memory properties for at least 9 weeks after endotoxemia

NK cells enriched from spleens of naïve mice were transferred into congenic mice $\left(1 \times 10^{6}\right.$ cells/recipient, intravenously) injected 9 weeks before with PBS (We9PBS) or LPS (10mg/kg, i.p.) (We9LPS). Recipient mice were challenged one day later with LPS $(10 \mathrm{mg} / \mathrm{kg})$ and sacrificed 6 hours later. Splenocytes were processed for flow cytometry to levels of intracellular IFN $\gamma$ in endogenous vs transferred NK cells. a) Data summary grouped before-after plots depicting percentages of IFN $\gamma+$ cells among endogenous We9PBS NK cells (black dots), transferred naive NK cells (blue dots) and endogenous We9LPS NK cells (red dots) at 6 hours after LPS rechallenge. NK cells were enriched from spleens of mice injected 9 weeks before with PBS (We9PBS) or LPS (10mg/kg, i.p.) (We9LPS) and were transferred into naïve congenic mice $\left(1 \times 10^{6}\right.$ cells/recipient, intravenously). Recipient mice were challenged and samples harvested as above. b) Data summary grouped before-after plots depicting percentages of IFN $\gamma+$ cells among endogenous naive NK cells (blue dots), transferred We9PBS NK cells (black dots) and transferred We9LPS NK cells (red dots) at 6 hours after LPS rechallenge. NK cells were enriched from spleens of We9PBS and LPS mice and stimulated in vitro with cytokines $(10 \mathrm{ng} / \mathrm{ml})$ or LPS $(100 \mathrm{ng} / \mathrm{ml})$ overnight. Quantification of c) IFN $\gamma$ expression by NK cells and total levels of d) IFN $\gamma$ in respective conditions. Dots represent individual mice. Data are representative of one experiment out of 3 repeats with $n \geq 4$ mice/group. $n s$, not significant, $* * * p<0.001$. Wilcoxon paired test comparing D14SIn, D14LPS and naïve cell values under respective conditions (a-b) and MannWhitney test comparing D14PBS and D14LPS values (c-d). 


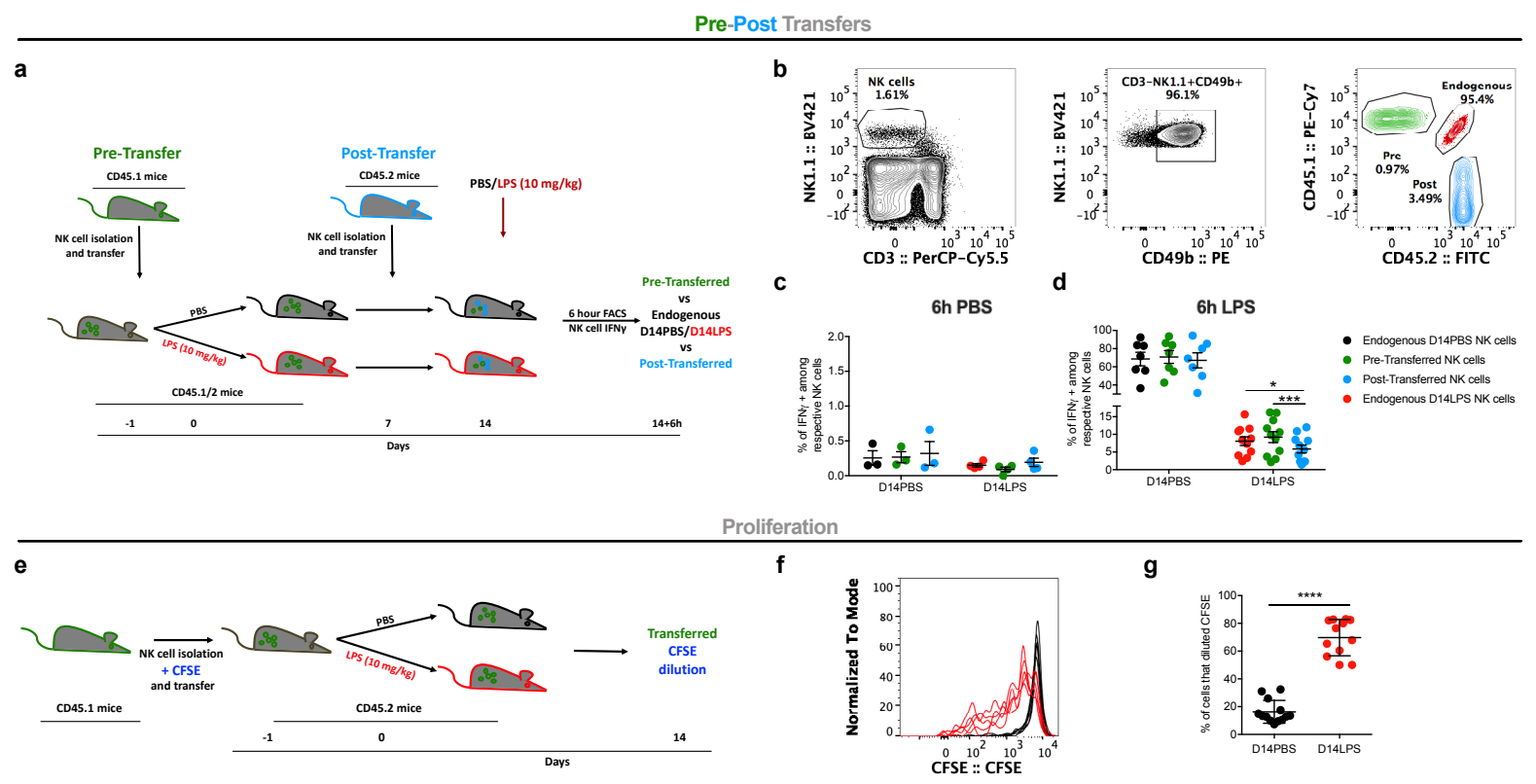

Fig. 4 Memory-like cells derive from NK cells that experienced endotoxemia

NK cells enriched from spleens of CD45.1 mice were Pre-Transferred into CD45.1/2 mice $\left(1 \times 10^{6}\right.$ cells/recipient, intravenously), one day before recipients were injected with PBS or LPS (10mg/kg, i.p.). Naïve CD45.2 enriched NK cells were Post-Transferred into the CD45.1/2 mice, 7 days after PBS/LPS injection. On day 14 with CD45.1/2 D14PBS and D14LPS mice were rechallenged with LPS and sacrificed 6 hours later to assess intracellular IFN $\gamma$ levels in endogenous, pre- and post-transferred NK cells. a) Experimental transfer scheme b) Representative gating strategy c) Data summary scatter plots depicting percentages of IFN $\gamma+$ cells among endogenous CD45.1/2 D14PBS NK cells (black dots), PreTransferred CD45.1 NK cells (green dots), Post-Transferred CD45.2 (blue dots) and endogenous CD45.1/2 D14LPS NK cells (red dots) at 6 hours after PBS or d) LPS re-challenge. Enriched CD45.1 naïve NK cells were labeled with CFSE and transferred into CD45.2 mice one day before PBS or LPS $(10 \mathrm{mg} / \mathrm{kg}$, i.p.) injection. On day 14, spleens were harvested from D14PBS and D14LPS mice, and stained for transferred NK cell identification in order to assess CFSE dilution. e) Experimental transfer scheme f) Representative overlay histogram showing CFSE dilution of CD45.1 transferred cells in D14PBS (black lines) and D14LPS (red lines). g) Data summary showing percentages of cells that diluted CFSE (gated on all cells before the highest intensity peak). Dots represent individual mice. Data are representative of one experiment out of 3 repeats with $\mathrm{n} \geq 5$ mice/group. ns, not significant, ${ }^{*} \mathrm{p}<0.05$, *** $\mathrm{p}<0.001$. Wilcoxon paired test comparing D14Sln, D14LPS and naïve cell values under respective conditions. 
a

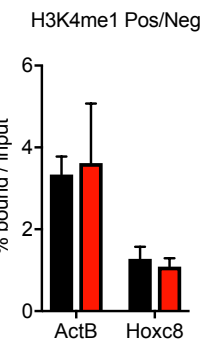

b

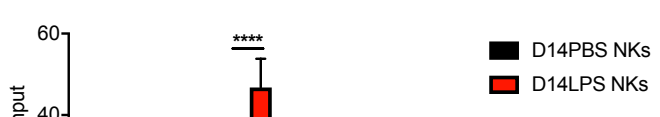

c
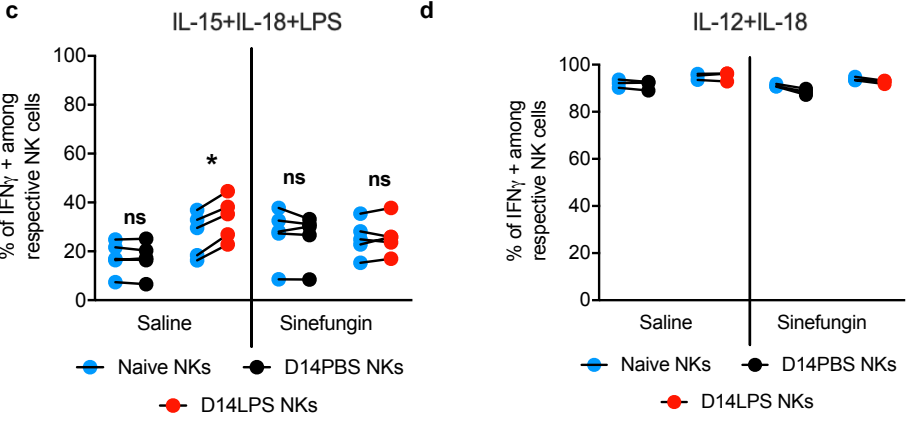

e

H3K4me1

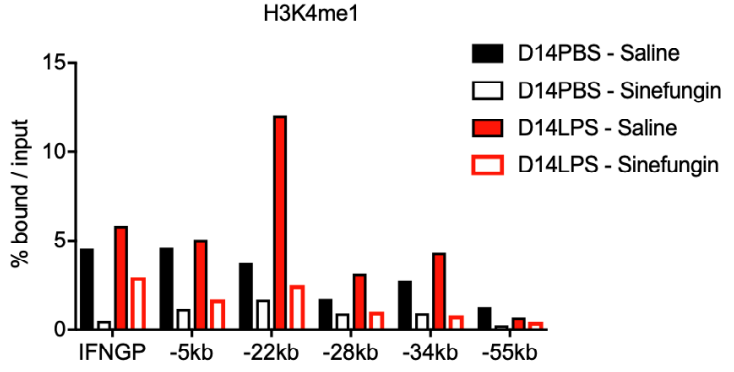

Fig. 5 Histone methylation maintains NK cell memory after endotoxemia

NK cells were isolated from spleens of mice injected 14 days before with PBS (D14PBS) or LPS $\left(10 \mathrm{mg} / \mathrm{kg}\right.$, i.p.) (D14LPS). Approximately $3 \times 10^{6}$ highly purified NK cells were fixed and chromatin was extracted and sheared. ChIP for $\mathrm{H} 3 \mathrm{~K} 4 \mathrm{mel}$ and $\mathrm{H} 3 \mathrm{~K} 4 \mathrm{me} 3$ was perfomed and resulting positive and input fractions of the chromatin were amplified by PCR for the indicated targets. a) Enrichment percentage for H3K4me1 pulldown on positive (ActB) and negative (Hox8) controls. b) Enrichment percentage for H3K4me1 pulldown on different regions of the ifng locus. $\mathbf{c}$ and d) Mice were injected with PBS or LPS $(10 \mathrm{mg} / \mathrm{kg}$, i.p.). On day 2 , mice received either Saline or Sinefungin $(10 \mathrm{mg} / \mathrm{kg})$. On day 14 , NK cells were higly purified from mice D14PBS or D14LPS NK cells that were treated with Saline or Sinefungin. NK cells from each group were mixed with enriched naïve congenic NK cells and stimulated overnight in the indicated conditions. Cells were stained for flow cytometry analysis of intracellular IFN $\gamma$ levels. Data summary grouped before-after plots depicting percentages of IFN $\gamma+$ cells among naïve (blue dots), D14PBS (black dots) and D14LPS (red dots) NK cells. Treatment groups underneath graph. e) ChIP for H3K4me1 on chromatin of NK cells purified from D14PBS-Saline, D14PBS-Sinefungin, D14LPSSaline, D14LPS-Sinefungin mice. Enrichment percentage for H3K4mel pulldown on different regions of the ifng locus for each group is showed. Data are representative of one experiment out of at least 2 repeats with $\mathrm{n} \geq 3$ mice/group except for panel $\mathrm{d}$ which was performed once. ns, not significant, ${ }^{*} \mathrm{p}<0.05$, *** $\mathrm{p}<0.001$. Mann-Whitney test $(\mathrm{a}, \mathrm{b})$ and Wilcoxon paired test (c) comparing D14Sln, D14LPS and naïve cell values under respective conditions. 
a

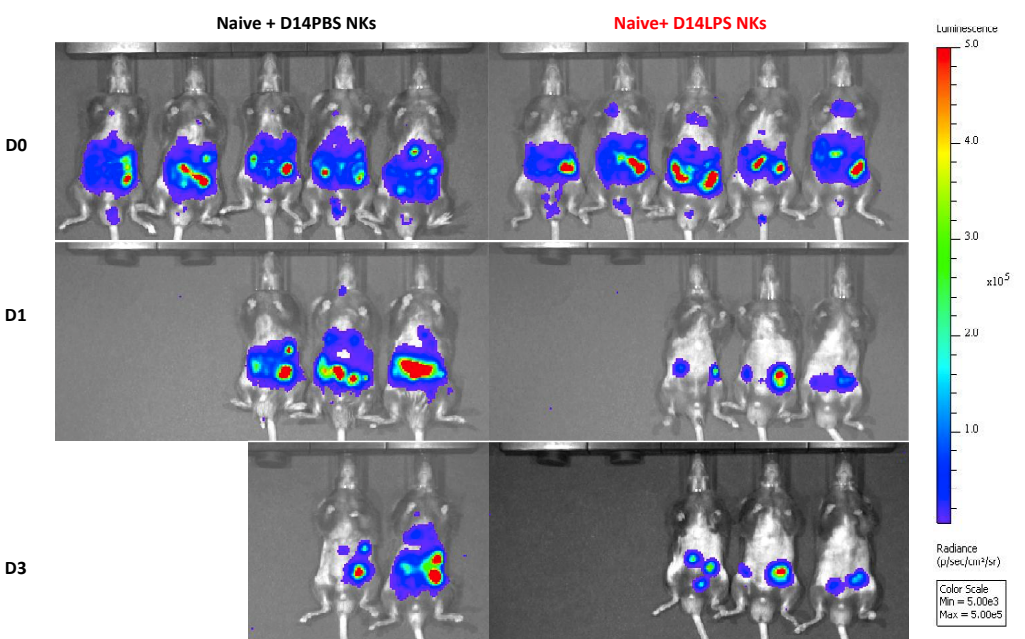

b Luminescence

c
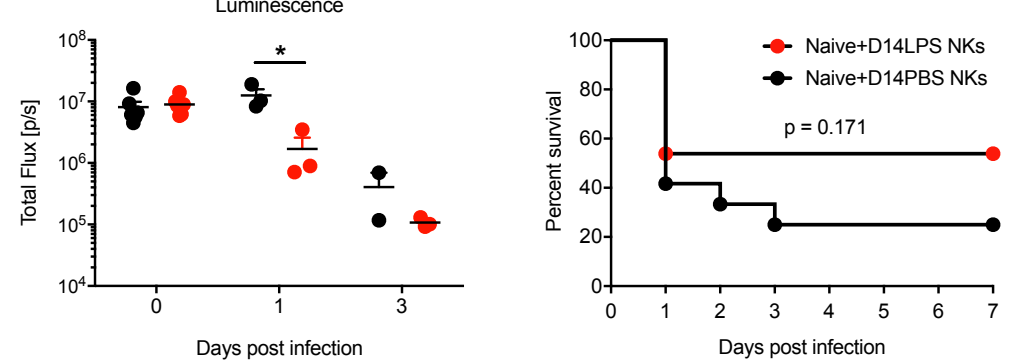

Fig. 6 Memory NK cells protect from bacterial infection

NK cells purified from spleens of mice that were injected 14 days before with PBS (D13PBS) or LPS $\left(10 \mathrm{mg} / \mathrm{kg}\right.$, i.p.) (D13LPS) were transferred into naive mice $\left(1 \times 10^{5}\right.$ cells/recipient, intravenously). Recipient mice were challenged two days later with a luminescent E. coli $\left(2 \times 10^{7} \mathrm{CFU} / \mathrm{mouse}\right)$ via intraperitoneal injection. Luminescent signal was acquired daily for 3 days post-infection, and survival was monitored for 7 days. a) Representative images of bacterial luminescent signal in challenged mice at day 1 and 3. b) Quantification of luminescent signal. c) Survival curve for challenged mice ( $\mathrm{n}=12$ 14/group). Data are representative of one experiment (panels a and b), or pooled results (panel c), from 2 repeats with $\mathrm{n} \geq 5$ mice/group. 
a
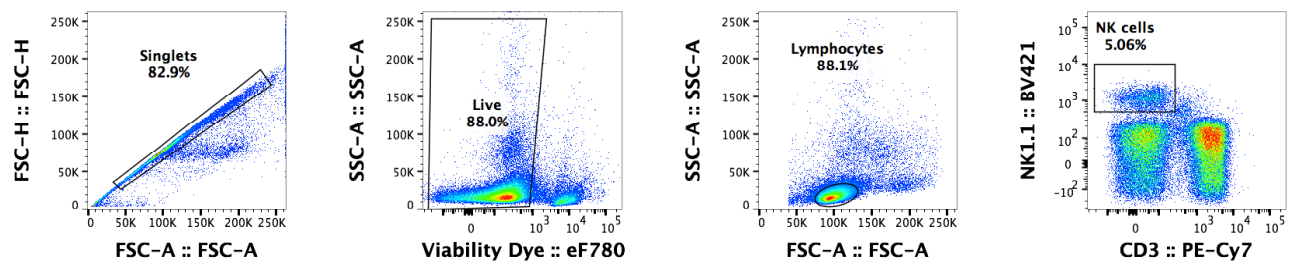

b
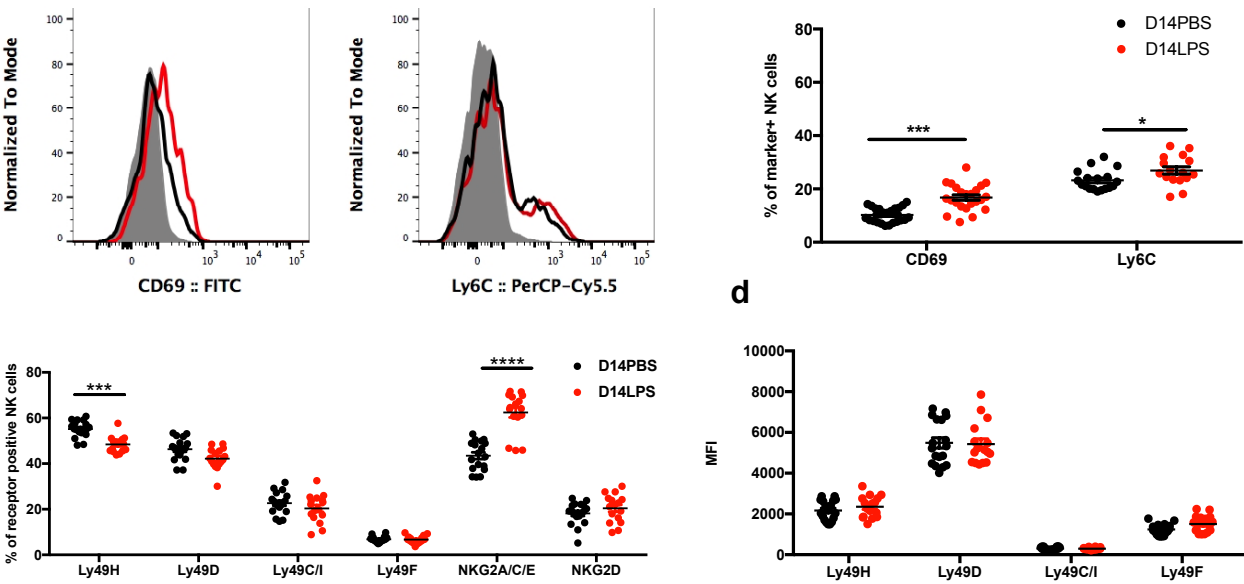

d

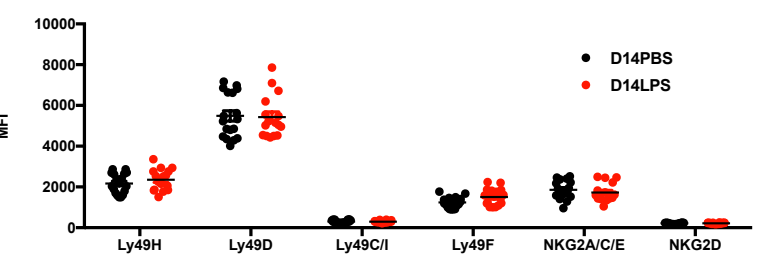

e

Baseline IFN $\gamma$

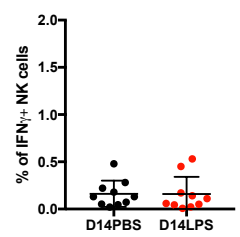

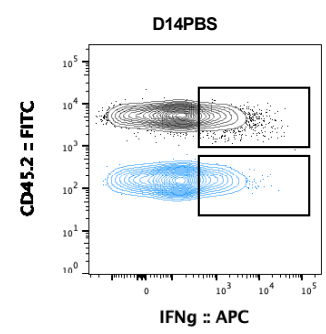

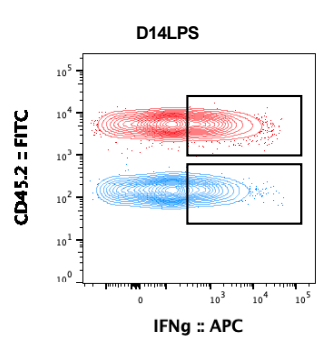

g

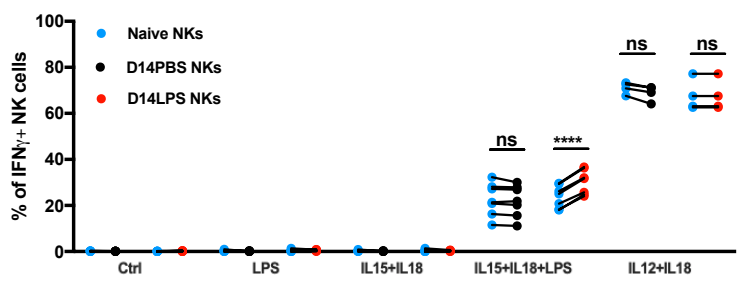

Fig. S1 NK cell gating strategy and NK receptor profiles after endotoxemia

Splenocytes were harvested from mice 2 weeks after they were intraperitoneally injected with PBS (D14PBS) or LPS (10mg/kg, i.p.) (D14LPS) and stained for NK cell identification (CD3-NK1.1+) and NK cell receptors. a) Gating strategy used for NK cell identification. b) representative overlay histograms and quantification of CD69 and Ly6C expression c) Data summary of NK cell expression of several NK cell receptors in percentages and d) intensity of expression. e) Quantification of ex-vivo IFN $\gamma$ staining. Higly purified CD45.2 D14PBS or D14LPS NKs were mixed with enriched naïve CD45.1 NKs and stimulated overnight in the indicated conditions. Cells were stained for flow cytometry analysis of intracellular IFN $\gamma$ levels. f) Representative contour plots of IFNg staining among the co-cultured NKs. g) Data summary grouped before-after plots depicting percentages of IFN $\gamma+$ cells among CD45.1 naïve (blue dots), CD45.2 D14PBS (black dots) and D14LPS (red dots) NKs. Dots represent individual mice except for panels e,f where they represent technical repeats from group-pooled samples. Horizontal lines represent mean values $+/$ - SEM. Data represent pooled values from $2-5$ repeats with $n \geq 5$ mice/group for $\mathrm{b}$ and $\mathrm{c}$, and one representative experiment out of 3 repeats with $\mathrm{n} \geq 5$ mice/group for panels e.f. ns, not significant, ${ }^{*} \mathrm{p}<0.05,{ }^{* * *} \mathrm{p}<0.001$. Mann-Whitney test comparing D14Sln and D14LPS values under respective conditions. 
a

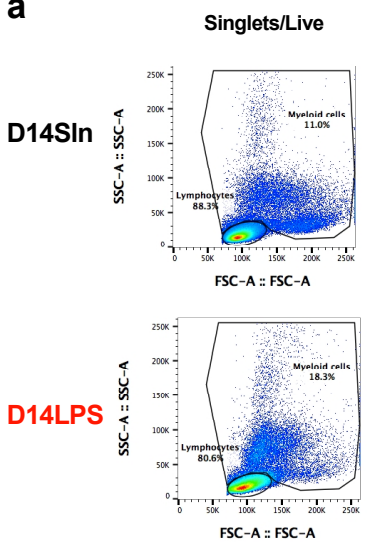

b

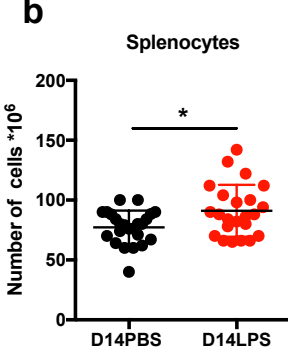

g

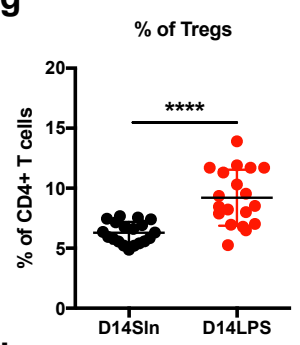

I

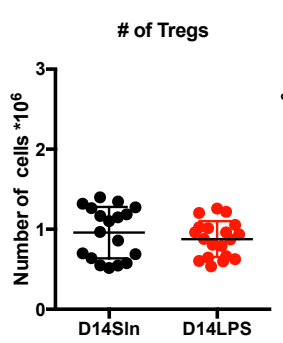

C

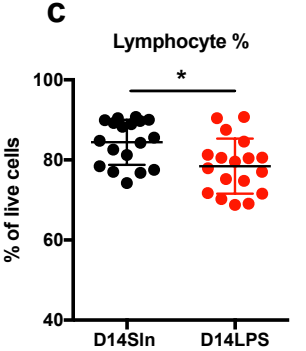

$\mathbf{h}$

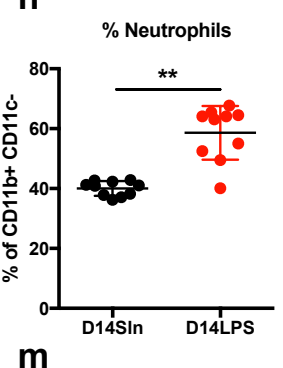

m

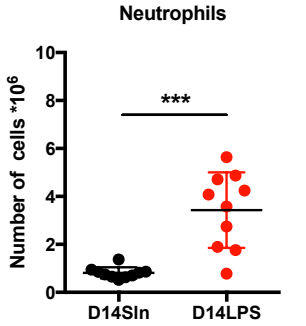

Lymphocytes
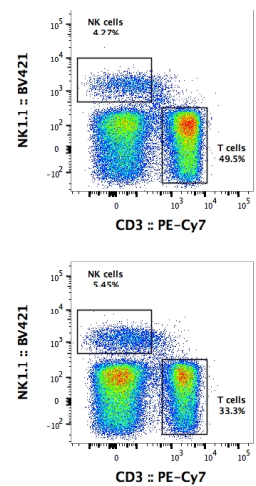

d

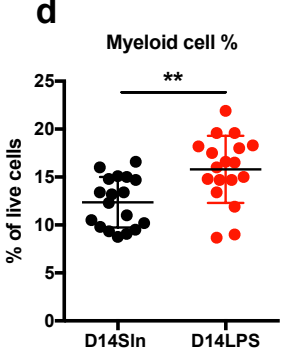

i
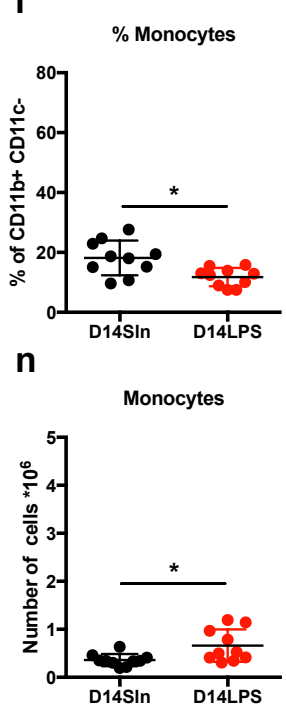

CD4+ $T$ cells
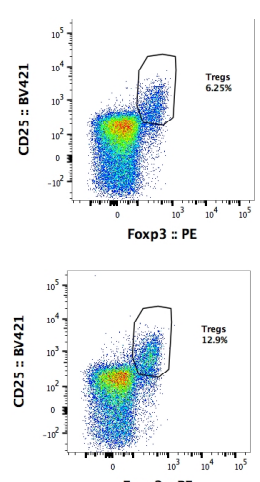

Tregs

e
Myeloid cells
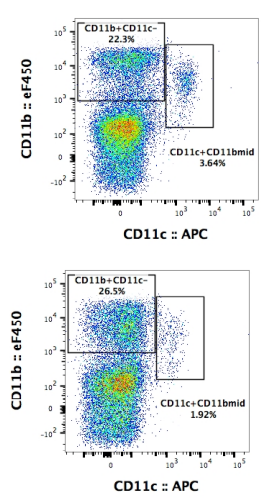

f
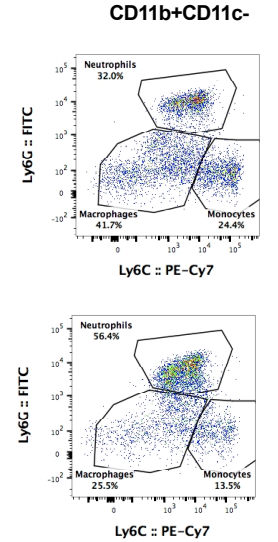

T cells
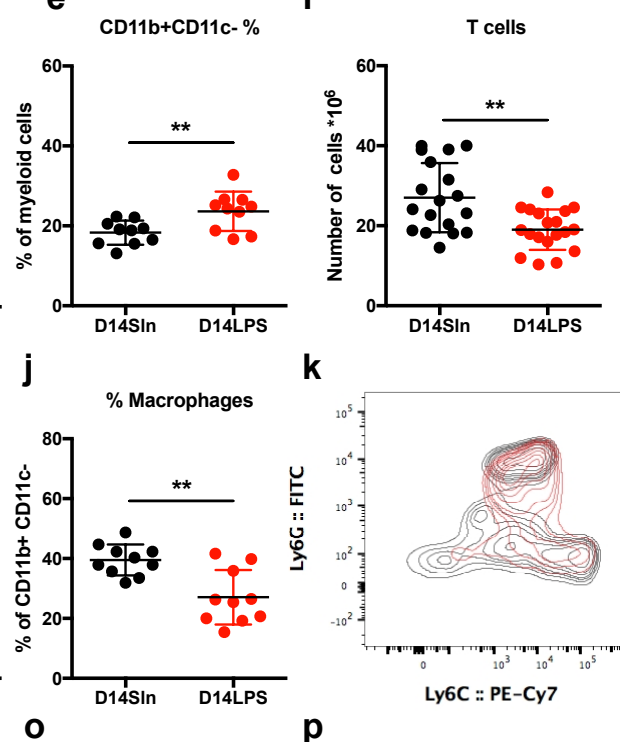

k

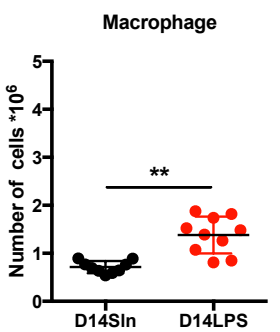

Fig. S2 Spleen environment is altered after endotoxemia

Splenocytes were harvested from mice 2 weeks after they were intraperitoneally injected with PBS (D14PBS) or LPS $\left(10 \mathrm{mg} / \mathrm{kg}\right.$, i.p.) (D14LPS). Cells were stained for identification of $\mathrm{T}$ cells $\left(\mathrm{NK} 1.1^{-} \mathrm{CD} 3^{+}\right)$, NK cells (NK1.1 $\left.1^{+} \mathrm{CD} 3\right)$, Tregs $\left(\mathrm{CD} 3{ }^{+} \mathrm{CD} 4^{+} \mathrm{CD} 25^{+} \mathrm{Foxp}^{+}\right)$, neutrophils (CD11b ${ }^{+} \mathrm{CD} 11 \mathrm{c}^{-}$Ly6G $\mathrm{G}^{\text {high }} \mathrm{Ly} 6 \mathrm{C}^{+}$), monocytes $\left(\mathrm{CD} 11 \mathrm{~b}^{+} \mathrm{CD} 11 \mathrm{c}^{-} \mathrm{Ly} 6 \mathrm{G}^{-} \mathrm{Ly} 6 \mathrm{C}^{\mathrm{high}}\right)$ and macrophages $\left(\mathrm{CD} 11 \mathrm{~b}^{+} \mathrm{CD} 11 \mathrm{c}^{-} \mathrm{Ly}_{6 \mathrm{G}^{-/+}} \mathrm{Ly}_{6 \mathrm{C}^{-/}}\right)$. (a) Representative dot plots depicting gating strategy and sample results from D14PBS and D14LPS splenocyte staining. (b) Total splenocyte numbers. (c) Lymphocyte and (d) myeloid cell percentages among live events. (e) Percentage of CD11b+CD11ccells among myeloid cells. (f) Total T cell numbers. (g) Percentage and (l) total number of Tregs. (h) Percentage and (m) total number of neutrophils. (i) Percentage and (n) total number of monocyte. (j) Percentage and (o) total number of macrophages. (k) Representative overlay contour plot of Ly6G/Ly6C profiles of myeloid cells from D14PBS (black) and D14LPS (red). (p) Quantification of staining intensity of CD11b and Ly6G on neutrophils. Horizontal lines represent mean values $+/$ - SEM. Dots represent individual mice. Data represent pooled values from 2-5 repeats with $n \geq 5$ mice/group ns, not significant, ${ }^{*} \mathrm{p}<0.05, * * * p<0.001$. Mann-Whitney test comparing D14PBS and D14LPS values under respective conditions. 

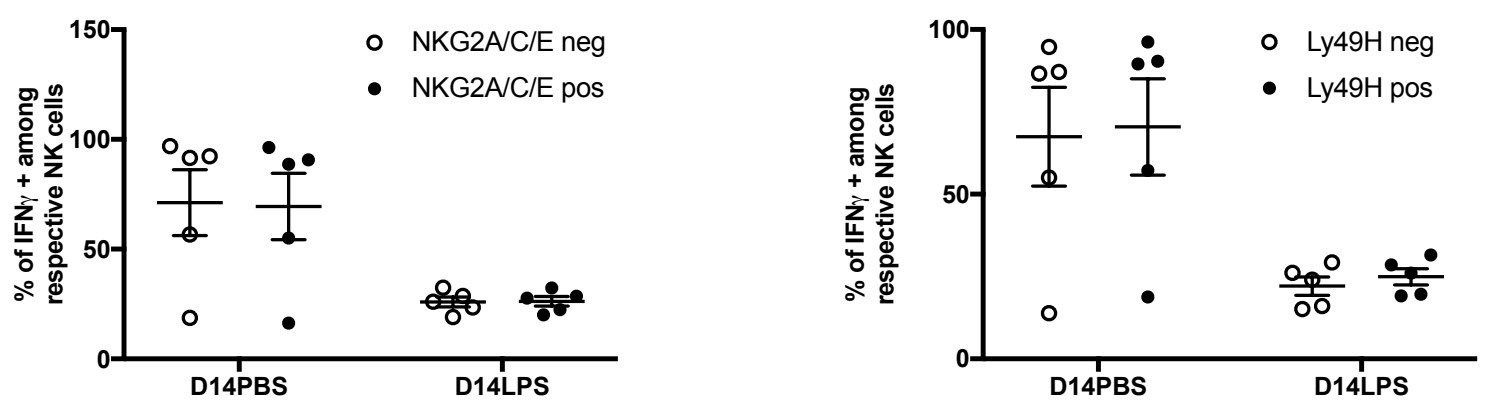

Fig. S3 NK cell response during LPS challenge in different sub-populations

Mice that were intraperitoneally injected 14 days in advance with PBS (D14PBS) or LPS (10mg/kg, i.p.) (D14LPS) were rechallenged with LPS (10mg/kg, i.p.). Six hours after re-challenge, splenocytes were processed for flow cytometry to identify NK cells positive and negative for NKG2A/C/E or Ly49H and the levels of intracellular IFN $\gamma$ in the respective sub-populations. a) Data summary of IFN $\gamma+$ percentages amonge the respective $\mathrm{NKG} 2 \mathrm{~A} / \mathrm{C} / \mathrm{E}$ positive or negative populations in D14PBS and LPS mice. b) Data summary of IFN $\gamma+$ percentages amonge the respective Ly49H positive or negative populations in D14PBS and LPS mice. Bars represent mean values +/- SEM. Dots represent individual mice. Data are representive of one out of 2 repeats $n=5$ mice/group ns, not significant. Mann-Whitney test comparing percentages of IFN $\gamma+$ among NKG2A/C/E or Ly49H positive to negative populations under respective conditions 
a

b
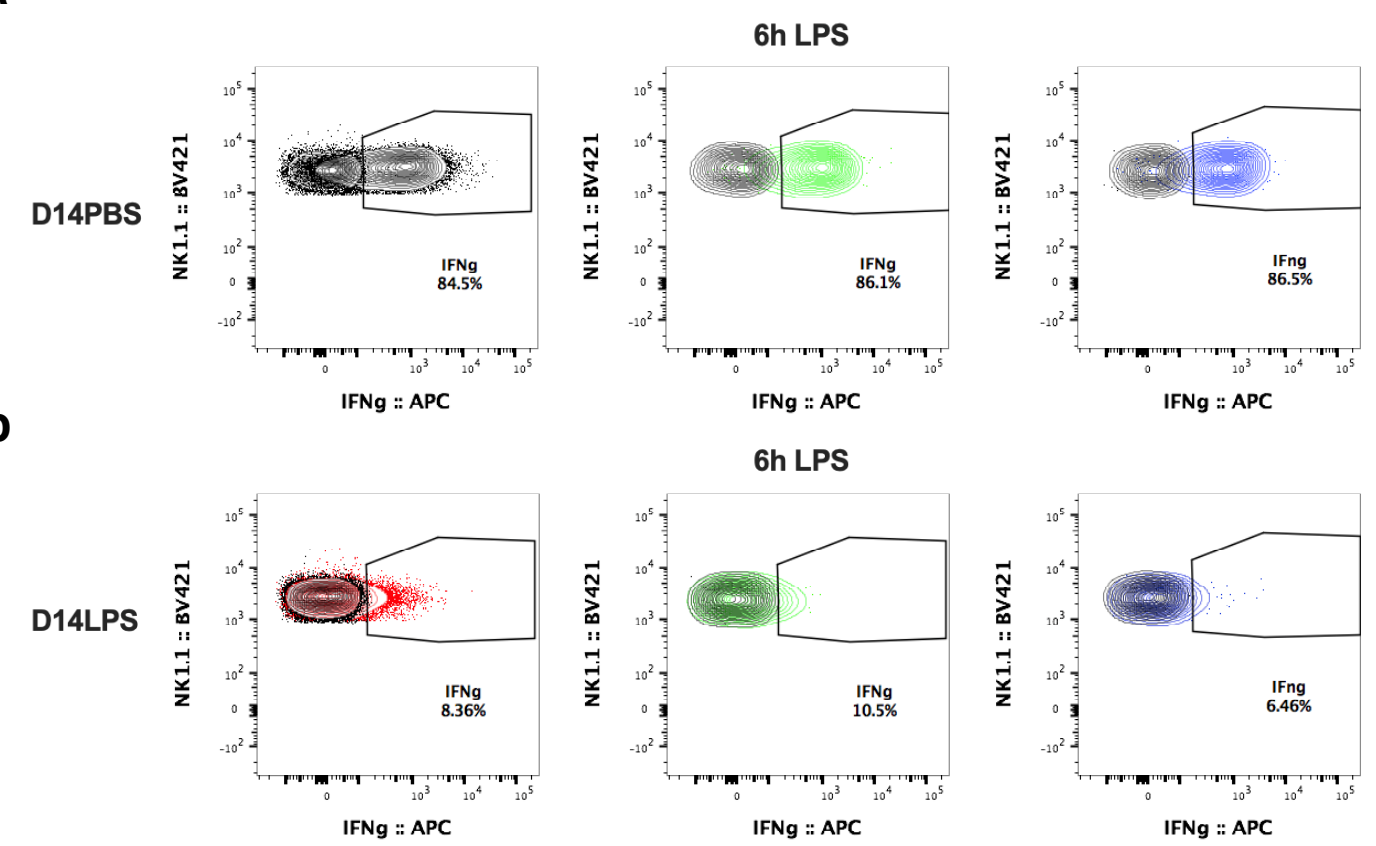

\section{c}

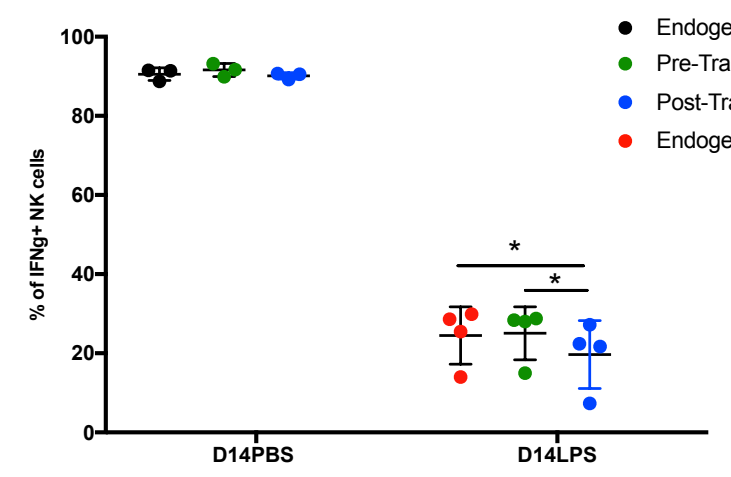

Fig. S4 NK cell responses in pre-post transfers

NK cells enriched from spleens of CD45.1 mice were Pre-Transferred into CD45.1/2 mice $\left(1 \times 10^{6}\right.$ cells/recipient, intravenously), one day before recipients were injected with PBS or LPS (10mg $/ \mathrm{kg}$, i.p.). Naïve CD45.2 enriched NK cells were Post-Transferred into the CD45.1/2 mice, 7 days after PBS/LPS injection. On day 14 with CD45.1/2 D14PBS and D14LPS mice were rechallenged with LPS and sacrificed 6 hours later to assess intracellular IFN $\gamma$ levels in endogenous, pre- and post-transferred NK cells. a) Representative results depicting percentages of IFN $\gamma+$ cells among endogenous CD45.1/2 D14PBS NKs (black), Pre-Transferred CD45.1 NK cells (green), PostTransferred CD45.2 (blue) b) and endogenous CD45.1/2 D14LPS NK cells (red) at 6 hours after LPS rechallenge. NK cells enriched from spleens of CD45.2 TLR2/4KO mice were Pre-Transferred into WT CD45.1/2 mice (1x10 ${ }^{6}$ cells/recipient, intravenously), one day before recipients were injected with PBS or LPS (10mg $/ \mathrm{kg}$, i.p.). Naïve CD45.1 enriched NK cells were Post-Transferred into the CD45.1/2 mice, 7 days after PBS/LPS injection. On day 14 with WT CD45.1/2 D14PBS and D14LPS mice were rechallenged with LPS and sacrificed 6 hours later to assess intracellular IFN $\gamma$ levels in endogenous, pre- and post-transferred NK cells. c) Data summary depicting percentages of IFN $\gamma+$ cells among endogenous WT CD45.1/2 D14PBS NKs (black dots), Pre-Transferred TLR2/4KO CD45.2 NK cells (green dots), Post-Transferred WT CD45.1 (blue dots) and endogenous WT CD45.1/2 D14LPS NK cells (red dots) at 6 hours after LPS rechallenge. Dots represent individual mice. Data are representative of one experiment out of 2 repeats with $n=3$-4mice/group. $n s$, not significant, ${ }^{*} p<0.05$. Wilcoxon paired test comparing Pre, Post and Endogenous NK cells under respective conditions. 
Table S1 ChIP primer sequences and antibodies

Primer

Sequence

IFNGP

5'CGAGGAGCCTTCGATCAGGT3'

5'GGTCAGCCGATGGCAGCTA3'

$-5 \mathrm{~Kb} \mathrm{CNS}$

5'CTAAGATGGTTCCCTGCCCA3'

5'CACTTGACATCTATCTATTCCTGCAGA3'

$-22 \mathrm{~kb}$ CNS

5'CCAGGACAGAGGTGTTAAGCCA3'

5'GCAACTTCTTTCTTCTCAGGGTG3'

$-28 \mathrm{~kb}$ CNS

5'TGGGAAGAAGTCCAAATGGG3'

5'TGCTTATTGGCTGGTCTAGCC3'

$-34 \mathrm{~kb}$ CNS

5'GGTATGCATCATCCCGGG3'

5'TGGCCTGTCTTCAGAAGTTTGC3'

$-55 \mathrm{~kb} \mathrm{CNS}$

5'GGCTTCCTGTCATTGTTTCCA3'

5'CAGAGCCATGGGATGACTGA3'

Actb

5'TGAGGTACTAGCCACGAGAGAG3'

5'TGAGGTACTAGCCACGAGAGAG3'

Hoxc8

5'CGTCGTCCCATTACCGAAAT3'

5'TTTCGGAAGCCACTTTGATTG3'

Antibodies

H3K4me3 Rabbit polyclonal antibody (C15410003 ; Diagenode)

H3K4me1 Rabbit polyclonal antibody (C15410194; Diagenode)

H3 Rabbit polyclonal antibody (ab1791; Abcam)

IgG Rabbit 